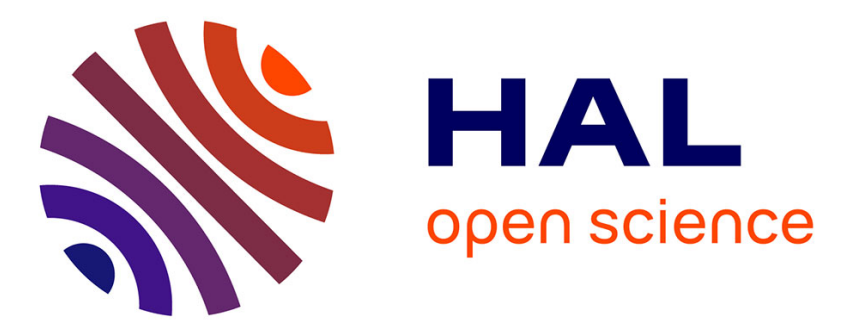

\title{
An innovative phase transition modeling for reproducing cavitation through a five-equation model and theoretical generalization to six and seven-equation models \\ Maria Giovanna Rodio, Rémi Abgrall
}

\section{- To cite this version: \\ Maria Giovanna Rodio, Rémi Abgrall. An innovative phase transition modeling for reproducing cavitation through a five-equation model and theoretical generalization to six and seven-equation models. [Research Report] RR-8664, INRIA Bordeaux; INRIA. 2015. hal-01100706v2}

\section{HAL Id: hal-01100706 \\ https://hal.inria.fr/hal-01100706v2}

Submitted on 15 Jan 2015

HAL is a multi-disciplinary open access archive for the deposit and dissemination of scientific research documents, whether they are published or not. The documents may come from teaching and research institutions in France or abroad, or from public or private research centers.
L'archive ouverte pluridisciplinaire $\mathbf{H A L}$, est destinée au dépôt et à la diffusion de documents scientifiques de niveau recherche, publiés ou non, émanant des établissements d'enseignement et de recherche français ou étrangers, des laboratoires publics ou privés. 
An innovative phase transition modeling for reproducing cavitation through a five-equation model and theoretical generalization to six and seven-equation models

Maria Giovanna Rodio, Remi Abgrall

RESEARCH REPORT $\mathrm{N}^{\circ} 8664$ January 2015 



\title{
Inzía
}

\section{An innovative phase transition modeling for reproducing cavitation through a five-equation model and theoretical generalization to six and seven-equation models}

\author{
Maria Giovanna Rodio, Remi Abgrall \\ Project-Teams Cardamom \\ Research Report n 8664 - January 2015 - 36 pages
}

\begin{abstract}
This work is devoted to model the phase transition for two-phase flows with a mechanical equilibrium model. First, a five-equation model is obtained by means of an asymptotic development starting from a non-equilibrium model (seven-equation model), by assuming a singlevelocity and a single pressure between the two phases, and by using the Discrete Equation Method (DEM) for the model discretization. Then, a splitting method is applied for solving the complete system with heat and mass transfer, i.e., the solution of the model without heat and mass transfer terms is computed and, then, updated by supposing a heat and mass exchange between the two phases. Heat and mass transfer is modeled by applying a thermo-chemical relaxation procedure allowing to deal with metastable states.

The interest of the proposed approach is to preserve the positivity of the solution, and to reduce at the same time the computational cost. Moreover, it is very flexible since, as it is shown in this paper, it can be extended easily to six (single velocity) and seven-equation models (non-equilibrium model).

Several numerical test-cases are presented, i.e. a shock-tube and an expansion tube problems, by using the five-equation model coupled with the cavitation model. This enables us to demonstrate, using the standard cases for assessing algorithms for phase transition, that our method is robust, efficient and accurate, and provides results at a lower CPU cost than existing methods. The influence of heat and mass transfer is assessed and we validate the results by comparison with experimental data and to the existing state-of-art methods for cavitation simulations.
\end{abstract}

Key-words: Heat and mass transfer, two-phase flows, Discrete Equation Method (DEM), sevenequation model, six-equation model, five-equation model, cavitation. 


\section{Un modèle de transition innovant pour simuler la cavitation à travers un modèle à 5 équations, et généralisation aux modèles à 6 et 7 équations}

Résumé : Ce travail est focalisé sur un modèle de transition de phase pour des écoulements diphasiques avec un modèle d'équilibre mécanique. Premièrement, un modèle à 5 équations est obtenu avec un développement asymptotique à partir d'un modèle à 7 équations, en supposant une seule vitesse et pression pour les deux phases, et en utilisant une méthode DEM (Discrete Equation Method) pour la discrétisation du modèle. De plus, une méthode de splitting est appliquée pour résoudre le système complet avec transfert de masse et de chaleur. Donc, la solution du modèle sans transfert de masse et de chaleur est calculée et mise à jour en supposant un transfert de masse et de chaleur. Le transfert de masse et chaleur est modélisé en appliquant une procédure de relaxation thermo-chimique permettant de traiter des états metastables. L'intért de cette approche est de préserver la positivité de la solution, et de réduire le coût de calcul. De plus, elle est très flexible et peut facilement être étendue à des modèles à 6 et 7 équations. Plusieurs cas-tests sont présentés, un tube à choc et une tube de détente, en utilisant un modèle à 5 équations couplé avec un modèle de cavitation. Cela permet de démontrer que notre méthode est robuste, précise et fournit des résultats avec un coût de calcul réduit par rapport aux méthodes existantes. L'influence du transfert de masse et de chaleur est estimée en comparant les données expérimentales avec des résultats existants en littérature.

Mots-clés : transferts de masse et de chaleur, écoulements diphasiques, DEM, modèles à 7 équations, modèles à 6 équations, modèles à 5 équations, cavitation. 


\section{Contents}

1 Introduction

2 The five-equation models with heat and mass transfer
3

5

3 The cavitation model for the five-equation model: a splitting method 7

3.1 Step 1: Numerical solution of a five-equation model with mechanical relaxation without heat and mass $t$

3.2 Step 2: Numerical solution of the temporal ODEs with heat and mass transfer terms 12

3.2.1 Stiff thermo-chemical solver for computing $Q$ and $\dot{Y} \ldots 14$

3.2 .2 Definition of $A, B, A^{\prime}$ and $B^{\prime} \quad \ldots \ldots \ldots \ldots \ldots$

3.3 Step 3: Mass fraction and density positivity . . . . . . 18

\begin{tabular}{|lll}
4 & Thermodynamic closure & 20
\end{tabular}

4.1 Stiffened Gas EOS for pure fluid . . . . . . . . . . . . 20

$4.2 \quad$ SG EOS based mixture . . . . . . . . . . . . . . . . . . . . 20

4.3 Definition of $x_{k}$ and $\kappa_{k} \ldots \ldots \ldots \ldots \ldots \ldots$

5 Extension to six and seven-equation model 21

5.1 Modeling cavitation with the seven-equation model . . . . . . . . 21

5.1 .1 Step 1 for a seven-equation model . . . . . . . . . . . 21

5.1 .2 Step 2 for a seven-equation model $\ldots \ldots \ldots . . . . . .22$

5.1 .3 Step 3 for a seven-equation model . . . . . . . . . . . 24

5.1 .4 Determination of $E_{t} \ldots \ldots \ldots \ldots \ldots$. . . . . . . . 24

5.2 Modeling cavitation for a six-equation model . . . . . . . . 25

$\begin{array}{lll}6 & \text { Results } & 25\end{array}$

6.1 TC1: Two-phase shock tube with mass transfer . . . . . . . . . 26

6.2 TC2: Simoes-Moreira shock tube experiments . . . . . . . 26

6.3 TC3: two-phase expansion tube with mixture initial velocities $v= \pm 2 \mathrm{~m} / \mathrm{s} 28$

6.4 TC4: two-phase expansion tube with mixture initial velocities $v= \pm 500 \mathrm{~m} / \mathrm{s} 28$

$\begin{array}{lll}7 & \text { Conclusions and perspective } & 29\end{array}$

$\begin{array}{lll}8 & \text { Acknowledgement } & 29\end{array}$

\section{Introduction}

Modeling the heat and mass transfer between the phases is of utmost importance when simulating flows in fuel injectors, turbo-pumps, nozzles, etc. Usually, in some of these applications, cavitation could appear because of a strong liquid acceleration, yielding a strong rarefaction wave and hence with vapor/gas bubbles creation. In Fig. 1, the pressure-volume diagram is illustrated. The dashed line (from 'saturated liquid' to 'saturated vapor' in Fig. 1) is the saturation curve, where the two phases are characterized by the same pressure, temperature and chemical potential. Following 1, 2, let us assume the liquid phase evolves following the isotherm line (solid line), from the state A to the state B. If it is depressurized to a pressure below the corresponding saturation pressure of state $\mathrm{B}$, it can evaporates, moving on the isothermal line (arriving to C), up to the equilibrium condition in the gas monophasic region (for example in state E) (see 


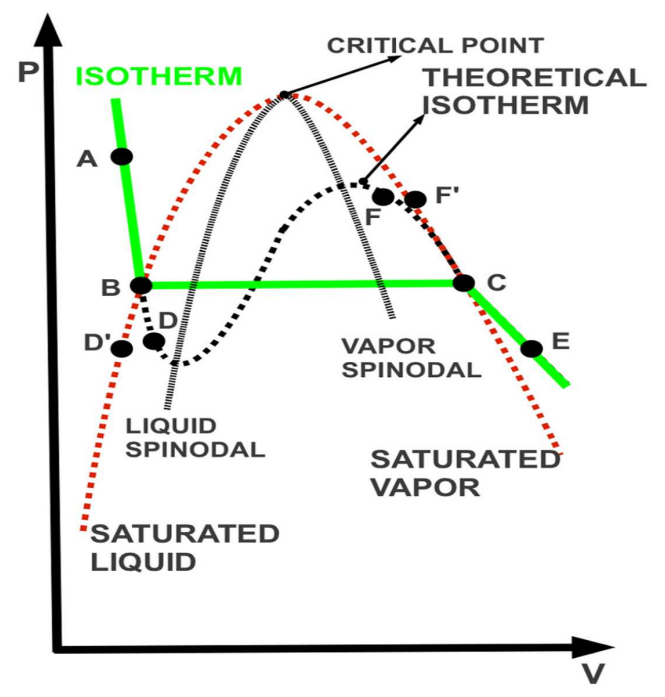

Figure 1: Pressure-volume diagram

[1] for more details). However, if the liquid undergoes a very strong rarefaction wave, it can evolves from the state A to a 'metastable' state (state D), i.e. a nonequilibrium state. This means that, in these conditions, the liquid phase releases its metastable energy, producing vapor and, thus, evolving in a new equilibrium state (for example in state E). The consequences of cavitation, such as noise, performance device reduction and wall corrosion, are extremely negative. For these reasons, this phenomenon requires a good theoretical comprehension and, as consequence, an accurate predictive physical model is required.

In the literature, several numerical models have been proposed for reproducing the two-phase flows. The most used are (i) the Bubbly Flow Models [3, 4, 5, 6, (ii) the Homogeneous Mixture Models [7, 8, 9, 10, 11] and (iii) the Multiphase Models [12, 13, 14, 15, 16. The main difference, among these families of models, consists in the assumptions for modeling the interaction and the behavior of the two phases.

In this paper, the focus is on the Interface diffuse Models. In one dimension, they consist in a system of three conservation equations for each phase and a transport equation for the vapor fraction. This system enables the explicit treatment of the mass, momentum and energy exchanges as transfer terms.

However, these models are numerically complex, and more recent works have proposed the introduction of relaxation procedures to reduce the system to five equations, ensuring the possibility of dealing with explicit mass and energy exchange [17, 18, 15. The most important reference about the treatment of heat and mass transfer terms, for the Interface Diffusive models, is the work of Saurel et al. [19. The authors proposed a phase transition modeling for a single velocity and single pressure model (five equations model) relying on a thermo-chemical relaxation. In fact, by obtaining the equality of temperature and chemical potential for the two phases, this relaxation assures the evolution 
of liquid phase from a metastable state to an equilibrium state on the saturation curve. During this evolution, the liquid phase releases mass in order to evaporate before assuming a new state on the saturation curve. Then, the authors proposed an original procedure to identify the liquid/vapor interface. In recent years, few works have been proposed for treating phase transition modeling in Interface Diffusive models [16, 19, 20, 21, 22, 23, 24]. Following the approach of [19, Zein at al. 20, proposed a heat and mass transfer model for the six equation system (single velocity model), based on three different relaxations of pressure, temperature and chemical potential, respectively. Recently, the same approach has been used by Wang al. in [23] and Daude et al. 22. Nevertheless, the original relaxation procedure of [19] and the successive modifications are affected by some numerical issues. In order to preserve the positivity of volume fraction and density, it might be necessary to integrate the system using time steps that are fractions of the hydrodynamic time step, thus yielding an increase of the computational cost (see [20]). Recently, Pelanti and Shyue 21] presented a new numerical treatment of heat and mass transfer terms for a six equation two-phase model. As in [25], they proposed, in an original way, a new procedure in order to guarantee the positivity of the system, based on the total entropy maximization, when the solution coming from the thermo-chemical relaxation step, is not physically admissible.

The first idea of the present paper is to couple the treatment of heat and mass transfer terms proposed in [19] for a five equation model, with the procedure for solution admissibility of [21], thus preserving the positivity of the solution and reducing consistently the computational cost. As a consequence, this method combines a good accuracy and a reduced computational cost, as demonstrated in the following by performing several comparisons with experimental data and other numerical results well-known in the literature. The second innovative point of this work is the theoretical extension of the proposed model to six and seven-equation models, thus showing its flexibility.

The paper is organized as follows. Section 2 presents the five-equation model with heat and mass transfer starting from a seven-equation one. Section 3 illustrates the main ingredients of the proposed approach for simulating cavitating flows. In Section 5, a theoretical extension of the cavitation model to the six and seven-equation model is presented and discussed. Section 6 illustrates several numerical results obtained with the five-equation model, displaying the robustness of the numerical method on the most used numerical configuration in the literature. In particular, the influence of heat and mass transfer is assessed and the results are validated by comparison with respect to the experimental data.

Finally, in Section 7, some conclusions and perspectives are drawn.

\section{The five-equation models with heat and mass transfer}

We introduce here the general formulation of a seven-equation model and the assumptions for yielding a five equations models, with heat and mass transfer 
20]:

$$
\begin{aligned}
& \frac{\partial \alpha_{1}}{\partial t}+v_{I} \frac{\partial \alpha_{1}}{\partial x}=\mu\left(P_{1}-P_{2}\right)+\eta Q+\frac{\rho}{\rho_{I}} \dot{Y} \\
& \frac{\partial\left(\alpha_{1} \rho_{1}\right)}{\partial t}+\frac{\partial\left(\alpha_{1} \rho_{1} v_{1}\right)}{\partial x}=\rho \dot{Y} \\
& \frac{\partial\left(\alpha_{1} \rho_{1} v_{1}\right)}{\partial t}+\frac{\partial\left(\alpha_{1} \rho_{1} v_{1}^{2}+\alpha_{1} P_{1}\right)}{\partial x}=P_{I} \frac{\partial \alpha_{1}}{\partial x}+\lambda\left(v_{2}-v_{1}\right)+v_{I} \rho \dot{Y} \\
& \frac{\partial\left(\alpha_{1} \rho_{1} E_{1}\right)}{\partial t}+\frac{\partial\left(\alpha_{1}\left(\rho_{1} E_{1}+P_{1}\right) v_{1}\right)}{\partial x}=P_{I} v_{I} \frac{\partial \alpha_{1}}{\partial x}+\lambda v_{I}\left(v_{2}-v_{1}\right)+ \\
& +\mu P_{I}\left(P_{1}-P_{2}\right)+Q+E_{I} \rho \dot{Y} \\
& \frac{\partial\left(\alpha_{2} \rho_{2}\right)}{\partial t}+\frac{\partial\left(\alpha_{2} \rho_{2} v_{2}\right)}{\partial x}=-\rho \dot{Y} \\
& \frac{\partial\left(\alpha_{2} \rho_{2} v_{2}\right)}{\partial t}+\frac{\partial\left(\alpha_{2} \rho_{2} v_{2}^{2}+\alpha_{2} P_{2}\right)}{\partial x}=P_{I} \frac{\partial \alpha_{2}}{\partial x}-\lambda\left(v_{2}-v_{1}\right)-v_{I} \rho \dot{Y} \\
& \frac{\partial\left(\alpha_{2} \rho_{2} E_{2}\right)}{\partial t}+\frac{\partial\left(\alpha_{2}\left(\rho_{2} E_{2}+P_{2}\right) v_{2}\right)}{\partial x}=P_{I} v_{I} \frac{\partial \alpha_{2}}{\partial x}-\lambda v_{I}\left(v_{2}-v_{1}\right)+ \\
& -\mu P_{I}\left(P_{1}-P_{2}\right)-Q-E_{I} \rho \dot{Y},
\end{aligned}
$$

where:

- $Q=\theta\left(T_{2}-T_{1}\right)$ is the heat transfer term, $T_{2}$ and $T_{1}$ are the liquid and the gas temperature, respectively

- $\dot{Y}=\nu\left(g_{2}-g_{1}\right)$ is the mass transfer term, $g_{2}$ and $g_{1}$ are the liquid and the gas Gibbs free energy, respectively.

The symbols $\rho_{k}, \alpha_{k}, v_{k}$ and $P_{k}$ represent the density, the volume fraction, the velocity and the pressure for each phase, respectively. The terms $\rho_{I}, E_{I}$, $P_{I}$ and $v_{I}$ are the density (see Eq. (10)), the energy (see Section [5.1.4), the pressure and the velocity (see Section 5.1.2) at the interface, respectively. Last, $\rho=\alpha_{1} \rho_{1}+\alpha_{2} \rho_{2}$ is the mixture density. The coefficients $\lambda=C / \varepsilon$ and $\mu=$ $C^{\prime} / \varepsilon$ represent the relaxation velocity parameter and the dynamic compaction viscosity. The coefficients $C$ and $C^{\prime}$ depend on the acoustic impedance of the fluids (see 26] for more details). These are the so-called relaxation terms, which yield a process inducing a pressure and velocity equilibrium.

The system (11) can be, also, expressed in vectorial form as follows:

$$
\frac{\partial U}{\partial t}+\frac{\partial}{\partial x} F(U)+B(U) \frac{\partial \alpha_{1}}{\partial x}=\frac{R(U)}{\varepsilon}+S(U)
$$

where

$$
\begin{gathered}
U=\left(\begin{array}{c}
\alpha_{k} \\
\alpha_{k} \rho_{k} \\
\alpha_{k} \rho_{k} v_{k} \\
\alpha_{k} \rho_{k} E_{k}
\end{array}\right), \quad F(U)=\left(\begin{array}{c}
0 \\
\alpha_{k} \rho_{k} v_{k} \\
\alpha_{k}\left(\rho_{k} v_{k}^{2}\right)+P_{k} \\
\alpha_{k}\left(\rho_{k} E_{k}+P_{k}\right) v_{k}
\end{array}\right) \\
B(U)=\left(\begin{array}{c}
v_{I} \\
0 \\
-P_{I} \\
-P_{I} v_{I}
\end{array}\right), \frac{R(U)}{\varepsilon}=\left(\begin{array}{c}
\mu\left(P_{k}-P_{k^{\star}}\right) \\
0 \\
\lambda\left(v_{k^{\star}}-v_{k}\right) \\
\lambda v_{I} \cdot\left(v_{k^{\star}}-v_{k}\right)-\mu P_{I}\left(P_{k}-P_{k}^{\star}\right) \\
-\mu\left(P_{1}-P_{2}\right)
\end{array}\right),{ }_{\text {Inria }}
\end{gathered}
$$




$$
S(U)=\left(\begin{array}{c}
\frac{\alpha_{1} \alpha_{2}}{\alpha_{2} \rho_{1} c_{1}^{2}+\alpha_{1} \rho_{2} c_{2}^{2}}\left(\frac{\Gamma_{1}}{\alpha_{1}}+\frac{\Gamma_{2}}{\alpha_{2}}\right) Q+\frac{\rho}{\rho_{I}} \dot{Y} \\
\rho \dot{Y} \\
v_{I} \rho \dot{Y} \\
Q+E_{I} \rho \dot{Y}
\end{array}\right) .
$$

In order to reduce the complexity of the system, mechanical equilibrium is considered. The equality of pressure and velocity can be obtained through a stiff mechanical relaxation of the system (1), as in 27, 21, 20, or through an asymptotic development, as in 28, 15, where relaxation parameters, $\lambda$ and $\mu$, are assumed as tending to infinity. This means that a very large interface area is assumed. After a Chapman-Enskog like expansion, the system (11) becomes:

$$
\begin{aligned}
& \frac{\partial \alpha_{1}}{\partial t}+v \frac{\partial \alpha_{1}}{\partial x}=K \frac{\partial v}{\partial x}+\eta Q+\frac{\rho}{\rho_{I}} \dot{Y} \\
& \frac{\partial \alpha_{1} \rho_{1}}{\partial t}+\frac{\partial\left(\alpha_{1} \rho_{1} v\right)}{\partial x}=\rho \dot{Y} \\
& \frac{\partial \alpha_{2} \rho_{2}}{\partial t}+\frac{\partial\left(\alpha_{2} \rho_{2} v\right)}{\partial x}=-\rho \dot{Y} \\
& \frac{\partial \rho v}{\partial t}+\frac{\partial\left(\rho v^{2}+P\right)}{\partial x}=0 \\
& \frac{\partial \rho E}{\partial t}+\frac{\partial(\rho E+P) v}{\partial x}=0
\end{aligned}
$$

where $E=e+\frac{1}{2} v^{2}$ is the mixture total energy and $e=\left(\alpha_{1} \rho_{1} e_{1}+\alpha_{2} \rho_{2} e_{2}\right) / \rho$ is the mixture internal energy. $K=\frac{\alpha_{1} \alpha_{2}\left(\rho_{2} c_{2}^{2}-\rho_{1} c_{1}^{2}\right)}{\left(\alpha_{1} \rho_{2} c_{2}^{2}+\alpha_{2} \rho_{1} c_{1}^{2}\right)}$. The unknowns are now $: \alpha_{1}$, $\rho_{k}, v, P, e_{k}, Q$ and $\dot{Y}$. This means that, for closing the system, two equations of state are used for defining the relation of energy as a function of pressure and density for both phases (see Section 4 ) and a cavitation model is used in order to define $\mathrm{Q}$ and $\dot{Y}$. This is detailed in Section 3 .

\section{The cavitation model for the five-equation model: a splitting method}

Now, let us consider the system (3). In order to compute a solution, a splitting procedure is adopted and the following three steps are solved:

- Step 1: compute the numerical solution of the hyperbolic part of system (3) without heat and mass transfer term source.

Three strategies are possible. The first one is to solve the five equation model directly, as in [19. The second possibility is to solve the seven equation model (system (11) without the heat and mass transfer terms and, then, to apply a mechanical relaxation in order to obtain the pressure and velocity equilibrium.

The third possible strategy is the one used in this work. It consists in following the procedure used in [14, 18, i.e. the discrete equation method (DEM) 29] applied to the system (11). An advantage to use the DEM method for (2) is an original way of addressing a important numerical 
challenge: the approximation of non-conservative term, when the velocity $\vec{v}$ and $K$ are simultaneously discontinuous (see [29, 18, 26] for more details).

Then, an asymptotic development is applied, at a discrete level, in order to impose to the semi-discrete system a mechanical relaxation. Finally, a final semi-discrete system is obtained, that is equivalent to discretize the five equation model (3).

- Step 2: update the solution of system (3), by solving the temporal ODEs system with the heat and mass transfer terms. This consists in applying a thermo-chemical relaxation that allows, from a metastable state, to attain a new equilibrium solution.

- Step 3: the thermo-chemical relaxation does not guarantee that the positivity of the solution be preserved, so a positivity check of the solution is performed.

As explained in Section 1 a thermo-chemical relaxation is required in order to compute a new equilibrium state.

By adopting the splitting method, it is assumed that the characteristic time of a mechanical relaxation, $1 / \mu$, is much smaller than the characteristic time scales $1 / \theta$ and $1 / \nu$ of heat and mass transfer terms, respectively. This means that thermal and chemical relaxation occur in a pressure equilibrium condition. In Fig. 2. the three steps are summarized in order to give a more complete vision of the global method.

In the following sections, the three steps are described in detail.

\subsection{Step 1: Numerical solution of a five-equation model with mechanical relaxation without heat and mass transfer}

The originality of this work is focused on the transition phase, thus for the step 1 , only the main lines of the numerical method, proposed in [18, are recalled.

We assume that at time $t$, the computational domain $\Omega$ is divided into the cells $\left.\mathcal{C}_{i}=\right] x_{i-1 / 2}, x_{i+1 / 2}[$. At a time $t=t+s$ (with $s$ small), the interface in $x_{i+1 / 2}$ moves at a velocity $\sigma_{i+1 / 2}$ and the interface in $x_{i-1 / 2}$ moves at a velocity $\sigma_{i-1 / 2}$. As a consequence, the cell $\mathcal{C}_{i}$ evolves in $\left.\overline{\mathcal{C}}_{i}=\right] x_{i-1 / 2}+s \sigma_{i-1 / 2}, x_{i+1 / 2}+$ $s \sigma_{i+1 / 2}[$ (see Fig. 3). The cell may be either smaller or larger than the original ones $\mathcal{C}_{i}$, depending on the signs of the velocities. So, the Godunov scheme [30] is no longer applied on the mesh cells, but on the modified and non-uniform cells constructed according to the position of the interface (see Fig. 31). Then, we introduce the characteristic function $X^{k}$ of the phase $\Sigma_{k}$. The function $X^{k}(x, t)$ is equal to 1 , if $x$ lies in the fluid $\Sigma_{k}$ at time $\mathrm{t}$ and 0 otherwise. Then, we denote with $F\left(U_{L}, U_{R}\right)$ the Godunov numerical flux between the states $U_{L}$ and $U_{R}$, and with $F^{\text {lag }}\left(U_{L}, U_{R}\right)$ the flux across the contact interface between the states $U_{L}$ and $U_{R}$ (see Fig. 3). The relation between the two fluxes is equal to :

$$
F^{l a g}\left(U_{L}, U_{R}\right)=F\left(U_{L R}^{+}\right)-\sigma\left(U_{L}, U_{R}\right) U_{L R}^{+}=F\left(U_{L R}^{-}\right)-\sigma\left(U_{L}, U_{R}\right) U_{L R}^{-},
$$

where the superscripts \pm denote the state on the right and on the left of the contact discontinuity, as in Fig. 3 


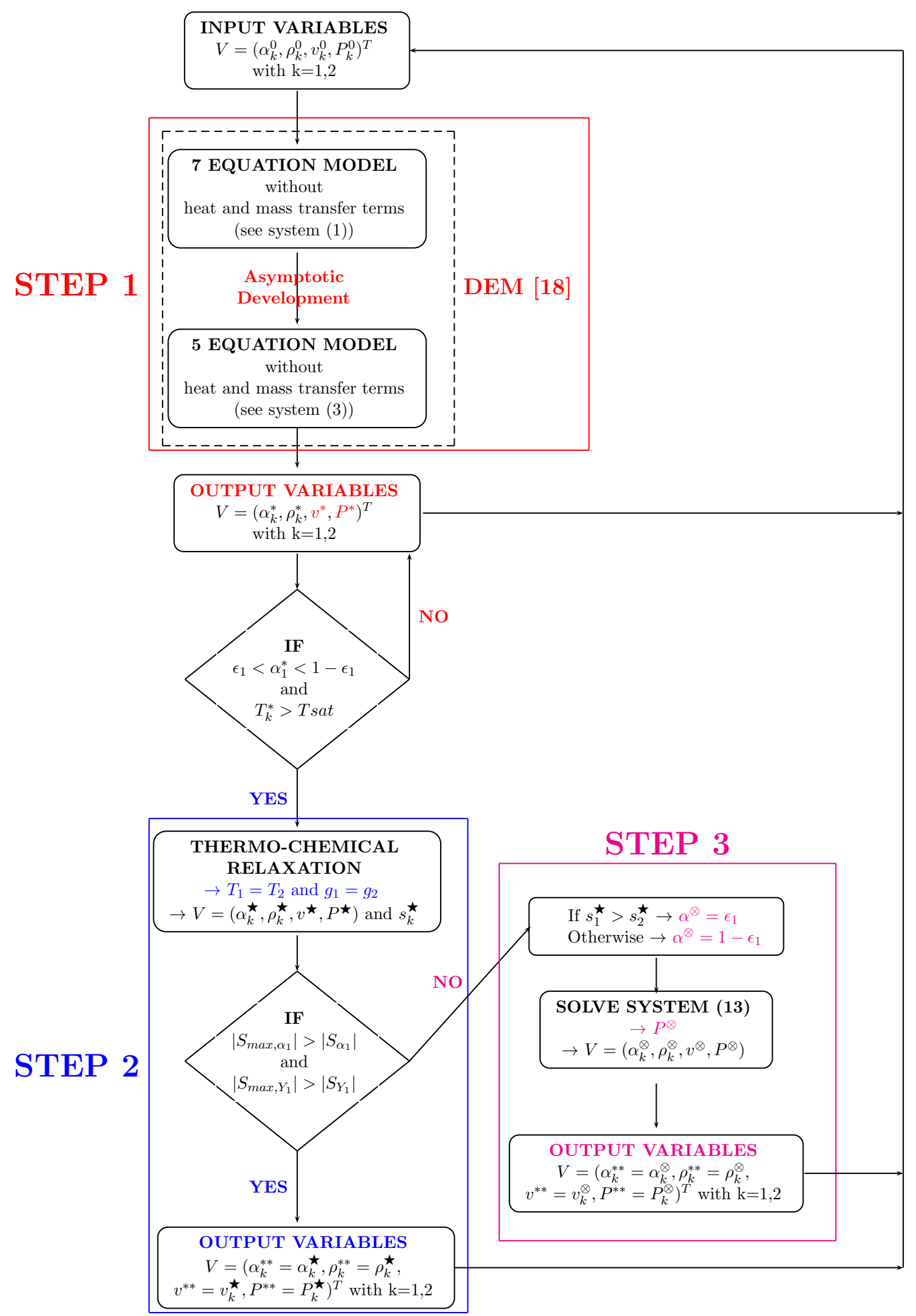

Figure 2: Sketch of the numerical scheme proposed in order to find the numerical solution of system (3).

$\mathrm{RR} \mathrm{n}^{\circ} 8664$ 

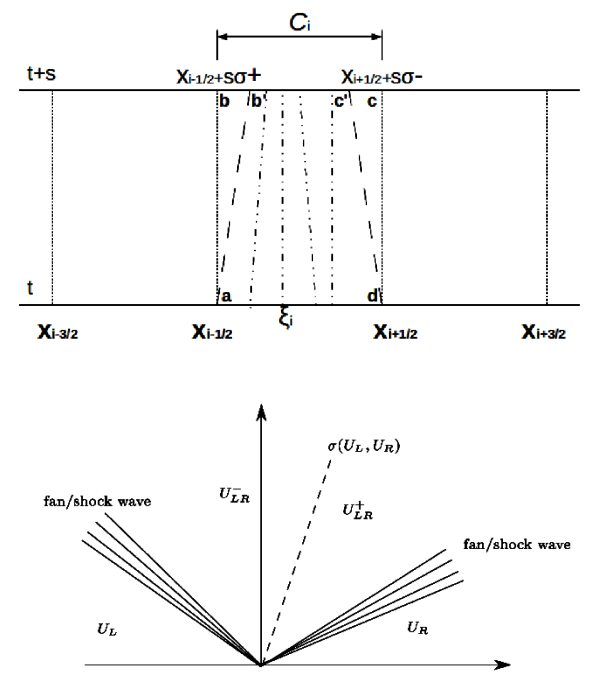

Figure 3: a) Subdivision of the computational domain. b) The various states in the Riemann problem between states $U_{L}$ and $U_{R}$.

In 29, a semi-discrete equation for the discretization of a seven-equation model (see Eq. (10) has been developed. Then, in [26, an asymptotic development has been applied on the numerical approximation of [29], obtaining the semi-discrete equation for the reduced model (see Eq. (3)). This last one is used in this work and its formulation is the following:

$$
\begin{aligned}
& \frac{\partial \alpha_{1}}{\partial t}=F T_{\alpha}\left(U_{1}\right)+\frac{\alpha_{1} \alpha_{2}}{\alpha_{2} \rho_{1} a_{1}^{2}+\alpha_{1} \rho_{2} a_{2}^{2}}\left\{\frac{F T_{\alpha}\left(U_{8}\right)}{\alpha_{2} \rho_{2} \chi_{2}}-\frac{u_{2} F T_{\alpha}\left(U_{7}\right)}{\alpha_{2} \rho_{2} \chi_{2}}+\right. \\
&+\frac{\frac{u_{2}^{2}}{2}-e_{2}-\rho_{2} \kappa_{2}}{\alpha_{2} \rho_{2} \chi_{2}} F T_{\alpha}\left(U_{6}\right)+\frac{\rho_{2}^{2} \kappa_{2} F T_{\alpha}\left(U_{1}\right)}{\alpha_{2} \rho_{2} \chi_{2}}-\frac{F T_{\alpha}\left(U_{4}\right)}{\alpha_{1} \rho_{1} \chi_{1}}+ \\
&\left.\quad+\frac{u_{1} F T_{\alpha}\left(U_{3}\right)}{\alpha_{1} \rho_{1} \chi_{1}}-\frac{\frac{u_{1}^{2}}{2}-e_{1}-\rho_{1} \kappa_{1}}{\alpha_{1} \rho_{1} \chi_{1}} F T_{\alpha}\left(U_{2}\right)-\frac{\rho_{1}^{2} \kappa_{1} F T_{\alpha}\left(U_{5}\right)}{\alpha_{1} \rho_{1} \chi_{1}}\right\} \\
& \frac{\partial \alpha_{1} \rho_{1}}{\partial t}=F T_{\alpha_{1} \rho_{1}}\left(U_{2}\right) \\
& \frac{\partial \alpha_{2} \rho_{2}}{\partial t}=F T_{\alpha_{2} \rho_{2}}\left(U_{6}\right) \\
& \frac{\partial \rho u}{\partial t}=F T_{\partial \rho u}\left(U_{3}\right)+F T_{\partial \rho u}\left(U_{7}\right) \\
& \frac{\partial \rho E}{\partial t}=F T_{\rho E}\left(U_{4}\right)+F T_{\rho E}\left(U_{8}\right)
\end{aligned}
$$

The vector $F T\left(U_{j}\right)=\left(F T_{\alpha}, F T_{\alpha_{1} \rho_{1}}, F T_{\alpha_{2} \rho_{2}}, F T_{\rho u}, F T_{\rho E}\right)$, with $j=1, \ldots, 8$, is defined by

$$
\begin{gathered}
F T\left(U_{j}\right)=\frac{1}{\triangle x} \mathcal{E}\left(X\left(x_{i+1 / 2}, t\right) F\left(U_{i+1 / 2}^{\star}\right)-X\left(x_{i-1 / 2}, t\right) F\left(U_{i-1 / 2}^{\star}\right)\right)+ \\
+\frac{1}{\triangle x}\left(\mathcal{E}\left([X]_{j=0}\right) F^{l a g}\left(U_{i}^{-}, U_{i-1}^{+}\right)-\mathcal{E}\left([X]_{j=N}\right) F^{l a g}\left(U_{i}^{+}, U_{i+1}^{-}\right)\right)
\end{gathered}
$$




\begin{tabular}{|c|c|c|}
\hline Flow Patterns & Jump indicator & Flux Indicator \\
\hline$\Sigma_{1}-\Sigma_{2}$ & {$[X]_{1,1}=0$} & $\left(\beta_{i+1 / 2}^{(1,2)}\right)=\left\{\begin{array}{lll}1 & \text { if } & \sigma\left(U_{i}^{l}, U_{i+1}^{r}\right) \geq 0 \\
-1 & \text { if } & \sigma\left(U_{i}^{l}, U_{i+1}^{r}\right)<0\end{array}\right.$ \\
\hline$\Sigma_{1}-\Sigma_{1}$ & {$[X]_{1,2}=\left\{\begin{array}{cc}-1 & \text { if } \sigma(1,2)>0 \\
0 & \text { otherwise }\end{array}\right.$} & 1 \\
\hline$\Sigma_{2}-\Sigma_{1}$ & {$[X]_{2,1}=\left\{\begin{array}{c}1 \text { if } \sigma(2,1)>0 \\
0 \quad \text { otherwise }\end{array}\right.$} & $\left(\beta_{i+1 / 2}^{(2,1)}\right)=\left\{\begin{array}{lll}1 & \text { if } & \sigma\left(U_{i}^{l}, U_{i+1}^{r}\right) \geq 0 \\
-1 & \text { if } & \sigma\left(U_{i}^{l}, U_{i+1}^{r}\right)<0\end{array}\right.$ \\
\hline$\Sigma_{2}-\Sigma_{2}$ & {$[X]_{2,2}=0$} & 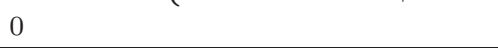 \\
\hline
\end{tabular}

Table 1: The various flow configurations at cell boundary $i+1 / 2$.

where $U_{i+1 / 2}^{\star}\left(\right.$ or $U_{i+1 / 2}^{\star}$ ) denotes the solution of Riemann problem between $U_{i}^{+}$and $U_{i+1}^{-}$(respectively, $U_{i-1}^{+}$and $\left.U_{i}^{-}\right)$. The quantities $[X]_{j=0}$ and $[X]_{j=N}$ are the jumps of $X$ at the beginning and at the end of the computational cell, respectively.

Note that the correspondence of the semi-discrete system (5) with the model (3) has been demonstrated in [26]. We notice that this method assumes initially two different thermodynamic states of phases, and at the end of the time step, the states have relaxed to a mechanical equilibrium. On the contrary, a direct discretization of the system (3) assumes directly the equality of initial pressure and velocity of the phases. Following [29, 26, the idea of DEM method is to avoid the introduction of an approximated estimation of fluxes expectancy, $\mathcal{E}(\cdot)$. So, this is estimated from the probability to find in two neighbor cells, the same phase or two different phases (see the "flow patterns" in the Table 1). Then, conservative and non-conservative terms of the system (5) can be developed assuming the four possible types of fluid phase discontinuities.

The terms of the vector $F T\left(U_{j}\right)$ of equation (5) can be computed as follows:

$$
\begin{aligned}
& \mathcal{E}\left(X\left(x_{i+\frac{1}{2}}, t\right) F\left(U_{i+\frac{1}{2}}^{\star}\right)\right)=\mathcal{P}_{i+\frac{1}{2}}\left(\Sigma_{1}, \Sigma_{1}\right) F\left(U_{i}^{(1)}, U_{i+1}^{(1)}\right)+ \\
& +\mathcal{P}_{i+\frac{1}{2}}\left(\Sigma_{1}, \Sigma_{2}\right)\left(\beta_{i+\frac{1}{2}}^{(1,2)}\right) F\left(U_{i}^{(1)}, U_{i+1}^{(2)}\right) \\
& +\mathcal{P}_{i+\frac{1}{2}}\left(\Sigma_{2}, \Sigma_{1}\right)\left(\beta_{i+\frac{1}{2}}^{(2,1)}\right) F\left(U_{i}^{(2)}, U_{i+1}^{(1)}\right) \\
& \mathcal{E}\left(X\left(x_{i-\frac{1}{2}}, t\right) F\left(U_{i-\frac{1}{2}}^{\star}\right)\right)=\mathcal{P}_{i-\frac{1}{2}}\left(\Sigma_{1}, \Sigma_{1}\right) F\left(U_{i-1}^{(1)}, U_{i}^{(1)}\right)+ \\
& +\mathcal{P}_{i-\frac{1}{2}}\left(\Sigma_{1}, \Sigma_{2}\right)\left(\beta_{i-\frac{1}{2}}^{(1,2)}\right) F\left(U_{i-1}^{(1)}, U_{i}^{(2)}\right) \\
& +\mathcal{P}_{i-\frac{1}{2}}\left(\Sigma_{2}, \Sigma_{1}\right)\left(\beta_{i-\frac{1}{2}}^{(2,1)}\right) F\left(U_{i-1}^{(2)}, U_{i}^{(1)}\right) \\
& \mathcal{E}\left([X]_{N} F^{l a g}\left(U_{i}^{N(w)}, U_{i+1}^{-}\right)\right)=\mathcal{P}_{1+1 / 2}\left(\Sigma_{1}, \Sigma_{2}\right)\left(\beta_{i+1 / 2}^{(1,2)}\right) F^{l a g}\left(U_{i}^{(1)}, U_{i+1}^{(2)}\right) \\
& -\mathcal{P}_{1+1 / 2}\left(\Sigma_{2}, \Sigma_{1}\right)\left(\beta_{i+1 / 2}^{(2,1)}\right) F^{l a g}\left(U_{i}^{(2)}, U_{i+1}^{(1)}\right) \\
& \mathcal{E}\left([X]_{0} F^{\text {lag }}\left(U_{i-1}^{+}, U_{i}^{0}\right)\right)=-\mathcal{P}_{1-1 / 2}\left(\Sigma_{1}, \Sigma_{2}\right)\left(\beta_{i-1 / 2}^{(1,2)}\right) F^{l a g}\left(U_{i-1}^{(1)}, U_{i}^{(2)}\right)+ \\
& +\mathcal{P}_{1-1 / 2}\left(\Sigma_{2}, \Sigma_{1}\right)\left(\beta_{i+1 / 2}^{(2,1)}\right) F^{l a g}\left(U_{i-1}^{(2)}, U_{i}^{(1)}\right)
\end{aligned}
$$

where $\chi_{k}$ and $\kappa_{k}$ are defined as follows:

$$
\chi_{k}=\left(\frac{\partial e_{k}}{\partial P_{k}}\right)_{\rho_{k}}, \kappa_{k}=\left(\frac{\partial e_{k}}{\partial \rho_{k}}\right)_{P_{k}},
$$

$\mathrm{RR} \mathrm{n}^{\circ} 8664$ 
$e_{k}$ is the phase internal energy.

It remains to evaluate the probabilities $\mathcal{P}_{i \pm 1 / 2}\left(\Sigma_{p}, \Sigma_{q}\right)$ (see [29]). For simplicity, we only show the final formulation for $i+1 / 2$ :

$$
\begin{array}{ll}
\mathcal{P}_{i+1 / 2}\left(\Sigma_{1}, \Sigma_{1}\right)=\min \left(\alpha_{i}^{(1)}, \alpha_{i+1}^{(1)}\right), & \mathcal{P}_{i+1 / 2}\left(\Sigma_{2}, \Sigma_{1}\right)=\max \left(\alpha_{i}^{(2)}-\alpha_{i+1}^{(2)}, 0\right) \\
\mathcal{P}_{i+1 / 2}\left(\Sigma_{1}, \Sigma_{2}\right)=\min \left(\alpha_{i}^{(2)}, \alpha_{i+1}^{(2)}\right), & \mathcal{P}_{i+1 / 2}\left(\Sigma_{1}, \Sigma_{2}\right)=\max \left(\alpha_{i}^{(1)}-\alpha_{i+1}^{(1)}, 0\right)
\end{array}
$$

where $\Sigma_{k}$ indicates the phase, with $k=1,2$.

The numerical flux $F(U)$ is approximated via an approximate Riemann solver which can define the contact speed $\sigma\left(U_{L}, U_{R}\right)$, allowing to define also the Lagrangian flux $F^{\text {lag }}$ (see Eq. (44)). In this paper, we have used the relaxation solver 31] for all computations (see 26] for more details). A second order has been applied by means of a predictor-corrector approach that is an extension to a multiphase flows of the MUSCL method shown in 32. It has been extensively explained in [29]. The time step is constrained by

$$
\left|\lambda_{\max }\right| \frac{\Delta x}{\Delta t} \leqslant \frac{1}{2}
$$

The semi-discrete equation (5) is solved in order to find the solution of the hyperbolic system with mechanical equilibrium. This strategy allows to start from two completely different thermodynamic states as in the seven-equation models, but finally, to find the mechanical equilibrium solution of a five equation model.

We denote with superscript " 0 " the initial quantities at time $t=0$ and with superscript $\star$ the quantities computed by solving Step 1. In Table 2, the input and output quantities of the first step are reported. In order to close the system (3), an equation of state (EOS) for each phase and for the mixture is used (see Section 4, and for more details Ref. [14]).

\begin{tabular}{c|c}
\hline \multicolumn{2}{c}{ Step 1 } \\
\hline \hline IN variables & OUT variables \\
\hline$\alpha_{1}^{0}\left(\alpha_{2}^{0}=1-\alpha_{1}^{0}\right)$ & $\alpha_{1}^{\star}\left(\alpha_{2}^{\star}=1-\alpha_{1}^{\star}\right)$ \\
$\rho_{1}^{0}$ and $\rho_{2}^{0}$ & $\rho_{1}^{\star}$ and $\rho_{2}^{\star}$ \\
$v_{1}^{0}$ and $v_{2}^{0}$ & $v^{\star}$ \\
$P_{1}^{0}$ and $P_{2}^{0}$ & $P^{\star}$ \\
$e_{1}^{0}$ and $e_{2}^{0}$ & $e_{1}^{\star}$ and $e_{2}^{\star}$ \\
$T_{1}^{0}$ and $T_{2}^{0}$ & $T_{1}^{\star}$ and $T_{2}^{\star}$ \\
$g_{1}^{0}$ and $g_{2}^{0}$ & $g_{1}^{\star}$ and $g_{2}^{\star}$ \\
\hline
\end{tabular}

Table 2: Variables used in the Step 1.

\subsection{Step 2: Numerical solution of the temporal ODEs with heat and mass transfer terms}

After considering the hydrodynamic evolution, let us focus now on the numerical treatment of heat and mass transfer terms. The following ODEs equation system 
allows to let evolve the solution, considering the new source terms:

$$
\begin{array}{rlrl}
\frac{\partial \alpha_{1}}{\partial t} & =\eta Q+\frac{\rho}{\rho_{I}} \dot{Y}:=S_{\alpha_{1}} & \\
\frac{\partial \alpha_{1} \rho_{1}}{\partial t} & =\rho \dot{Y}:=S_{Y_{1}} & \\
\frac{\partial \alpha_{2} \rho_{2}}{\partial t} & =-\rho \dot{Y}:=-S_{Y_{1}} & & \\
\frac{\partial \rho v}{\partial t} & =0, & \frac{\partial \rho E}{\partial t}=0 .
\end{array}
$$

where: $Q=\theta\left(T_{2}-T_{1}\right)$ and $\dot{Y}=\nu\left(g_{2}-g_{1}\right)$. The Gibbs free energy (chemical potential), $g_{k}$, are defined for a stiffened gas equation of state, as follows:

$$
g_{k}\left(P, T_{k}\right)=\left(\gamma_{k} C v_{k}-q^{\prime}\right) T_{k}-C v_{k} T_{k} \log \left(\frac{T_{k}^{\left(\gamma_{k}\right)}}{\left(P+P_{\infty, k}\right)^{\gamma_{k}-1}}\right) .
$$

The interface density has been defined in [19] and it has been determined assuming an isentropic behavior of acoustic waves, as follows:

$$
\rho_{I}=\frac{\frac{\rho_{1} c_{1}^{2}}{\alpha_{1}}+\frac{\rho_{2} c_{2}^{2}}{\alpha_{2}}}{\frac{c_{1}^{2}}{\alpha_{1}}+\frac{c_{2}^{2}}{\alpha_{2}}}
$$

Parameters $\theta$ and $\nu$ are the thermal and chemical relaxation parameters, respectively.

To identify the liquid/vapor interface, the method proposed in [19] and then in 20, 21] is used. In particular, the relaxation parameters $\theta$ and $\nu$ are set to zero far from the interfaces, and they are taken as infinite in order to fulfill equilibrium interface conditions with mass transfer:

$$
\theta, \nu= \begin{cases}+\infty & \text { if } \epsilon \leq \alpha_{1} \leq 1-\epsilon \\ 0 & \text { otherwise }\end{cases}
$$

where $\epsilon$ is a very low value. In order to define this value, we first remember that a limit of this model is that a phase can not completely disappear. This means that in all cells, the two phases always coexist. Anyway, a cell is considered filled by a pure fluid, when its volume and mass fraction is equal to $1-\epsilon_{1}$, with typically $\epsilon_{1}=10^{-8}$. In other words, in all cells, the gas phase can have a volume fraction value included in $\epsilon_{1}<\alpha_{g}<1-\epsilon_{1}$ and the liquid phase a complementary volume fraction, $\alpha_{l}=1-$ alph $_{g}$, in order to assure that also the other phase exists.

As a consequence, for identifying the interface, $\epsilon=10^{-6}$ is taken, and the interface corresponds to mixture cells when volume fractions range is between $\epsilon$ and $1-\epsilon$. If $\epsilon$ and $\epsilon_{1}$ are too close, evaporation may occur too early and not only in the interfacial zone.

Moreover, we impose that the mass transfer is allowed only if metastable state is fulfilled, i.e. if $T_{k}>T_{\text {sat }}\left(P^{\star}\right)$. The saturation temperature has been defined in Section 4

Let us denote with superscript ' $\star \star \star$ ' the final quantities computed by solving Step 1. In Table (3), the input and output quantities of the second step and the 
variables that are not influenced by the heat or mass transfer are reported. In fact, from system (11), it is clear that thanks to mass conservation, the mixture density is constant $\left(\rho^{\star \star}=\rho^{\star}\right)$ and thus, the velocity $\left(v^{\star \star}=v^{\star}\right)$ and the mixture total and internal energy $\left(E^{\star \star}=E^{\star}\right.$ and $\left.e^{\star \star}=e^{\star}\right)$.

Remembering that $\alpha_{2}=1-\alpha_{1}$, the unknowns of the system (9) are $\alpha_{1}$, $\rho_{k}, v, P, e_{k}, Q$ and $\dot{Y}$. Then, in order to close the system, two ingredients are required : (i) an equation of state for defining the energy as function of pressure and density for both phases (see Section 4) (ii) a thermo-chemical relaxation for finding $Q$ and $\dot{Y}$. The thermo-chemical relaxation is described in the following sections (Sections 3.2.1 and 3.2.2).

\begin{tabular}{c|c|c}
\hline \multicolumn{2}{c}{ Step 2 (Only at the interface: if $\epsilon_{1}<\alpha_{1}<1-\epsilon_{1}$ and $\left.T_{k}>T_{\text {sat }}\left(P^{\star}\right)\right)$} \\
\hline \hline IN variables & OUT variables & Constant MIXTURE variables \\
\hline$\alpha_{1}^{\star}\left(\alpha_{2}^{\star}=1-\alpha_{1}^{\star}\right)$ & $\alpha_{1}^{\star \star}\left(\alpha_{2}^{\star \star}=1-\alpha_{1}^{\star \star}\right)$ & \\
$\rho_{1}^{\star}$ and $\rho_{2}^{\star}$ & $\rho_{1}^{\star \star}$ and $\rho_{2}^{\star \star}$ & $\rho^{\star \star}=\rho^{\star}$ \\
$v^{\star}$ & $v^{\star \star}$ & $e^{\star \star}=e^{\star}$ \\
$P^{\star}$ & $P^{\star \star}$ & $E^{\star \star}=E^{\star}$ \\
$e_{1}^{\star}$ and $e_{2}^{\star}$ & $e_{1}^{\star \star}$ and $e_{2}^{\star \star}$ & \\
$T_{1}^{\star}$ and $T_{2}^{\star}$ & $T^{\star \star}$ & \\
$g_{1}^{\star}$ and $g_{2}^{\star}$ & $g^{\star \star}$ & \\
\hline
\end{tabular}

Table 3: Variables used in Step 2. $\rho=\left(\alpha_{1} \rho_{1}\right)+\left(\alpha_{2} \rho_{2}\right) ; \rho e=\left(\alpha_{1} \rho_{1} e_{1}\right)+\left(\alpha_{2} \rho_{2} e_{2}\right)$; $E=e+\frac{1}{2} v^{2}$.

\subsubsection{Stiff thermo-chemical solver for computing $Q$ and $\dot{Y}$}

It is well-known that, physically, the single phase in metastable state evolves in a new equilibrium liquid/vapor state with a heat and mass exchange. Thus, the mass transfer can stop, only when a new state on the saturation curve is attained, since the liquid and its vapor are in equilibrium. In order to estimate if the two phases are in equilibrium on the saturation curve, it should be assured that, in all time steps, the system achieves the temperature and Gibbs free energy equilibrium between the phases $\left(T_{1}=T_{2}\right.$ and $\left.g_{1}=g_{2}\right)$.

For solving this issue, a procedure similar to [19] is used. The temperature and the Gibbs time derivatives are computed, that can be written in terms of $\mathrm{Q}$ and $\dot{Y}$, using the mass equations and the energy mixture equation (see Section 3.2 .2 for details), as follows:

$$
\left\{\begin{array}{l}
\frac{\partial \Delta T}{\partial t}=A Q+B \dot{Y} \\
\frac{\partial \Delta g}{\partial t}=A^{\prime} Q+B^{\prime} \dot{Y}
\end{array}\right.
$$

where A, B, $A^{\prime}$ and $B^{\prime}$ should be defined. Assuming that thermodynamic equilibrium is reached at the end of each time step, the simplest numerical approximation of Eq. (12) can be written as follows:

$$
\frac{\partial \Delta T}{\partial t}=\frac{\Delta T^{n+1}-\Delta T^{n}}{\Delta t}=\frac{0-\Delta T^{n}}{\Delta t}=A^{n} Q^{n}+B^{n} \dot{Y}^{n}
$$




$$
\frac{\partial \Delta g}{\partial t}=\frac{\Delta g^{n+1}-\Delta g^{n}}{\Delta t}=\frac{0-\Delta g^{n}}{\Delta t}=A^{\prime n} Q^{n}+B^{\prime n} \dot{Y}^{n} .
$$

Thus, finally, we can determine the heat and mass transfer terms that are computed as follows :

$$
\left\{\begin{array}{l}
Q=-\frac{B^{\prime}}{A B^{\prime}-A^{\prime} B} \frac{(\Delta T)^{n}}{\Delta t}+\frac{B}{A B^{\prime}-A^{\prime} B} \frac{(\Delta g)^{n}}{\Delta t} \\
\dot{Y}=\frac{A^{\prime}}{A B^{\prime}-A^{\prime} B} \frac{(\Delta T)^{n}}{\Delta t}+\frac{A}{A B^{\prime}-A^{\prime} B} \frac{(\Delta g)^{n}}{\Delta t}
\end{array}\right.
$$

To compute $Q$ and $\dot{Y}, A, B, A^{\prime}$ and $B^{\prime}$ for a five-equation model should be provided. This permits to finalize Step 2. The definitions of these coefficients are provided in the following section.

\subsubsection{Definition of $A, B, A^{\prime}$ and $B^{\prime}$}

From classical thermodynamical identities, the temporal derivatives of temperature and Gibbs free energy differences can be written as follows :

$$
\begin{gathered}
\frac{\partial \Delta T}{\partial t}=\frac{\partial T_{2}}{\partial t}-\frac{\partial T_{1}}{\partial t} \\
\frac{\partial \Delta g}{\partial t}=\frac{\partial\left(h_{2}-T_{2} S_{2}\right)}{\partial t}-\frac{\partial\left(h_{1}-T_{1} S_{1}\right)}{\partial t}= \\
=\frac{1}{\rho_{2}} \frac{\partial P}{\partial t}-S_{2} \frac{\partial T_{2}}{\partial t}-\frac{1}{\rho_{1}} \frac{\partial P}{\partial t}+S_{1} \frac{\partial T_{1}}{\partial t}
\end{gathered}
$$

The temporal derivatives of temperatures and pressure can be defined thanks to mass conservation equations of each phase and to the mixture total energy conservation equation (9). From $\rho_{k}=\rho\left(P, T_{k}\right)$, the vapor mass conservation equation can be written as :

$$
\frac{\partial \alpha_{1} \rho_{1}}{\partial t}=\alpha_{1} \frac{\partial \rho_{1}}{\partial t}+\rho_{1} \frac{\partial \alpha_{1}}{\partial t}=\alpha_{1}\left(\left.\frac{\partial \rho_{1}}{\partial T_{1}}\right|_{P} \frac{\partial T_{1}}{\partial t}+\left.\frac{\partial \rho_{1}}{\partial P}\right|_{T_{1}} \frac{\partial P}{\partial t}\right)+\rho_{1} \frac{\partial \alpha_{1}}{\partial t}=\rho \dot{Y}
$$

If a stiffened gas equation of state is used (for the equation $29 \mathrm{c}$ ), it follows that:

$$
\left.\frac{\partial \rho_{1}}{\partial T_{1}}\right|_{P}=-\frac{\rho_{1}}{T_{1}} \quad \text { and }\left.\quad \frac{\partial \rho_{1}}{\partial P}\right|_{T_{1}}=\frac{1}{\left(\gamma_{1}-1\right) C v_{1} T_{1}}=\frac{\gamma_{1}}{c_{1}^{2}}
$$

where $c_{k}$ is the phase speed of sound. Thus, after some manipulations, the equation (15) becomes:

$$
\frac{\partial P}{\partial t}=\frac{c_{1}^{2}}{\alpha_{1} \gamma_{1}}\left(\rho \dot{Y}-\rho_{1} \frac{\partial \alpha_{1}}{\partial t}+\frac{\alpha_{1} \rho_{1}}{T_{1}} \frac{\partial T_{1}}{\partial t}\right) .
$$

The same procedure can be applied to liquid mass conservation equation of (9), obtaining:

$$
-\frac{\alpha_{2} \rho_{2}}{T_{2}} \frac{\partial T_{2}}{\partial t}+\frac{\alpha_{2} \gamma_{2}}{c_{2}^{2}} \frac{\partial P}{\partial t}-\rho_{2} \frac{\partial \alpha_{2}}{\partial t}=-\rho \dot{Y}
$$

Now, using (16) in (17), the partial derivative of liquid temperature can be obtained :

$$
\frac{\partial T_{2}}{\partial t}=\frac{T_{2}}{\alpha_{2} \rho_{2}}\left(\frac{D_{2}}{D_{1}} \frac{\alpha_{1} \rho_{1}}{T_{1}} \frac{\partial T_{1}}{\partial t}+C_{2} \dot{Y}-C_{1} \frac{\partial \alpha_{1}}{\partial t}\right)
$$


where:

$$
\begin{gathered}
D_{1}=\alpha_{1} \gamma_{1} c_{2}^{2} \\
D_{2}=\alpha_{2} \gamma_{2} c_{1}^{2} \\
C_{1}=\frac{D_{2} \rho_{1}+D_{1} \rho_{2}}{D_{1}} \\
C_{2}=\left(\frac{D_{2}+D_{1}}{D_{1}}\right) \rho
\end{gathered}
$$

Another equation is required to determine the partial derivative of vapor temperature. Remembering that in Step 2 the mixture $\rho, \rho v$ and $\rho E$ are constant, the following expression can be written:

$$
\frac{\partial \rho e}{\partial t}=e_{1} \frac{\partial \alpha_{1} \rho_{1}}{\partial t}+e_{2} \frac{\partial \alpha_{2} \rho_{2}}{\partial t}+\alpha_{1} \rho_{1} \frac{\partial e_{1}}{\partial t}+\alpha_{2} \rho_{2} \frac{\partial e_{2}}{\partial t}=0 .
$$

Since $\left(\partial \alpha_{1} \rho_{1} / \partial t=\rho \dot{Y}\right)$ and $\partial \alpha_{2} \rho_{2} / \partial t=-\rho \dot{Y}$ and by supposing that $e_{k}=$ $e\left(\rho_{k}, T_{k}\right)$, Eq. (19) can be written as:

$\left(e_{1}-e_{2}\right) \rho \dot{Y}+Y_{1} \rho\left(\left.\frac{\partial e_{1}}{\partial T_{1}}\right|_{\rho_{1}} \frac{\partial T_{1}}{\partial t}+\left.\frac{\partial e_{1}}{\partial \rho_{1}}\right|_{T_{1}} \frac{\partial \rho_{1}}{\partial t}\right)+Y_{2} \rho\left(\left.\frac{\partial e_{2}}{\partial T_{2}}\right|_{\rho_{2}} \frac{\partial T_{2}}{\partial t}+\left.\frac{\partial e_{2}}{\partial \rho_{2}}\right|_{T_{2}} \frac{\partial \rho_{2}}{\partial t}\right)=0$,

where $Y_{k}=\alpha_{k} \rho_{k} / \rho$. The Eq29b allows an easy computation of the partial derivatives, as follows:

$$
\begin{gathered}
\left.\frac{\partial e_{k}}{\partial T_{k}}\right|_{\rho_{k}}=C v_{k} \\
\left.\frac{\partial e_{k}}{\partial \rho_{k}}\right|_{T_{k}}=-\frac{P_{\infty, k}}{\rho_{k}^{2}} .
\end{gathered}
$$

Moreover, from the phase mass equations and the vapor volume fraction equation, we can determine $\partial \rho_{k} / \partial t$ as follows :

$$
\begin{gathered}
\frac{\partial \rho_{1}}{\partial t}=\rho \dot{Y}\left(\frac{1}{\alpha_{1}}-\frac{\rho_{1}}{\alpha_{1} \rho_{I}}\right)-\frac{\rho_{1}}{\alpha_{1}} \eta Q \\
\frac{\partial \rho_{2}}{\partial t}=-\rho \dot{Y}\left(\frac{1}{\alpha_{2}}-\frac{\rho_{2}}{\alpha_{2} \rho_{I}}\right)+\frac{\rho_{2}}{\alpha_{2}} \eta Q,
\end{gathered}
$$

where

$$
\eta=\frac{\alpha_{1} \alpha_{2}}{\alpha_{2} \rho_{1} c_{1}^{2}+\alpha_{1} \rho_{2} c_{2}^{2}}\left(\frac{\Gamma_{1}}{\alpha_{1}}+\frac{\Gamma_{2}}{\alpha_{2}}\right) .
$$

By replacing all derivatives in Eq. (20), we obtain:

$$
\begin{gathered}
\left\{\left(e_{1}-e_{2}\right)+\rho\left[\frac{P_{\infty, 2} Y_{2}}{\rho_{2}^{2}}\left(\frac{1}{\alpha_{2}}-\frac{\rho_{2}}{\alpha_{2} \rho_{I}}\right)-\frac{P_{\infty, 1} Y_{1}}{\rho_{1}^{2}}\left(\frac{1}{\alpha_{1}}-\frac{\rho_{1}}{\alpha_{1} \rho_{I}}\right)\right]\right\} \dot{Y}+ \\
+\left[Y_{1} \frac{P_{\infty, 1}}{\rho_{1} \alpha_{1}}-Y_{2} \frac{P_{\infty, 2}}{\rho_{2} \alpha_{2}}\right] \eta Q+Y_{1} C_{v_{1}} \frac{\partial T_{1}}{\partial t}+Y_{2} C_{v_{2}} \frac{\partial T_{2}}{\partial t}=(21) \\
=\theta_{1} \dot{Y}+Z_{1} \eta Q+Y_{1} C_{v_{1}} \frac{\partial T_{1}}{\partial t}+Y_{2} C_{v_{2}} \frac{\partial T_{2}}{\partial t}=0, \quad \text { Inria }
\end{gathered}
$$


where

$$
\theta_{1}=\left(e_{1}-e_{2}\right)+\rho\left[\frac{P_{\infty, 2} Y_{2}}{\rho_{2}^{2}}\left(\frac{1}{\alpha_{2}}-\frac{\rho_{2}}{\alpha_{2} \rho_{I}}\right)-\frac{P_{\infty, 1} Y_{1}}{\rho_{1}^{2}}\left(\frac{1}{\alpha_{1}}-\frac{\rho_{1}}{\alpha_{1} \rho_{I}}\right)\right]
$$

and

$$
Z_{1}=Y_{1} \frac{P_{\infty, 1}}{\rho_{1} \alpha_{1}}-Y_{2} \frac{P_{\infty, 2}}{\rho_{2} \alpha_{2}}
$$

We can replace, now, the partial derivative of liquid temperature $T_{2}$ (see Eq. 18):

$$
\begin{aligned}
\frac{\partial T_{1}}{\partial t}=\frac{T_{1} D_{1}}{Y_{1}\left(C_{v_{1}} T_{1} D_{1}+C_{v_{2}} T_{2} D_{2}\right)}\{-\dot{Y} & {\left[\theta_{1}+\frac{T_{2} C_{v_{2}}}{\rho}\left(C_{2}-\frac{\rho}{\rho_{I}}\right)\right] } \\
& \left.-Q \eta\left(Z_{1}-\frac{T_{2} C_{v_{2}}}{\rho} C_{1}\right)\right\} .
\end{aligned}
$$

Let us consider the system composed by the equations (16), (18) and (22):

$$
\begin{aligned}
& \frac{\partial P}{\partial t}=\frac{c_{1}^{2}}{\alpha_{1} \gamma_{1}}\left(\rho \dot{Y}-\rho_{1} \frac{\partial \alpha_{1}}{\partial t}+\frac{\alpha_{1} \rho_{1}}{T_{1}} \frac{\partial T_{1}}{\partial t}\right) \\
& \frac{\partial T_{2}}{\partial t}=\frac{T_{2}}{\alpha_{2} \rho_{2}}\left(\frac{D 2}{D 1} \frac{\alpha_{1} \rho_{1}}{T_{1}} \frac{\partial T_{1}}{\partial t}+C 2 \dot{Y}-C 1 \frac{\partial \alpha_{1}}{\partial t}\right) \\
& \frac{\partial T_{1}}{\partial t}=\frac{T_{1} D 1}{Y_{1}\left(C v_{1} T_{1} D_{1}+C v_{2} T_{2} D_{2}\right)}\left\{-\dot{Y}\left[\theta_{1}+\frac{T_{2} C v_{2}}{\rho}\left(C_{2}-\frac{\rho}{\rho_{I}}\right)\right]\right. \\
& \left.-Q \eta\left(Z 1-\frac{T_{2} C v_{2}}{\rho} C 1\right)\right\} \text {. }
\end{aligned}
$$

By replacing the temporal derivative of the vapor volume fraction $\left(\frac{\partial \alpha_{1}}{\partial t}=\right.$ $\left.\eta Q+\frac{\rho}{\rho_{I}} \dot{Y}\right)$, the temporal derivatives of the phase temperatures and pressure can be obtained as a function of $\dot{Y}$ and $Q$ :

$$
\begin{aligned}
& \frac{\partial T_{1}}{\partial t}=\xi\left\{-\dot{Y}\left[\theta_{1}+\frac{T_{2} C_{v_{2}}}{\rho}\left(C_{2}-\frac{\rho}{\rho_{I}}\right)\right]-Q \eta\left(Z_{1}-\frac{T_{2} C_{v_{2}}}{\rho} C_{1}\right)\right\} \\
& \frac{\partial T_{2}}{\partial t}=\frac{T_{2} D_{2} Y_{1}}{T_{1} D_{1} Y_{2}} \frac{\partial T_{1}}{\partial t}+\frac{T_{2}}{\alpha_{2} \rho_{2}}\left[\dot{Y}\left(C_{2}-C_{1} \frac{\rho}{\rho_{I}}\right)-C_{1} \eta Q\right] \\
& \operatorname{RR} \underset{n}{ } \frac{\partial P}{\partial \varphi^{6}}=\frac{c_{1}^{2} \rho_{1}}{\gamma_{1} T_{1}} \frac{\partial T_{1}}{\partial t}+\frac{c_{1}^{2}}{\alpha_{1} \gamma_{1}}\left[\dot{Y}\left(\rho-\rho_{1} \frac{\rho}{\rho_{I}}\right)-\rho_{1} \eta Q\right],
\end{aligned}
$$


where $\xi=\frac{T_{1} D 1}{Y_{1}\left(C_{v_{1}} T_{1} D_{1}+C_{v_{2}} T_{2} D_{2}\right)}$. Finally, substituting (24) in (14), it follows:

$$
\begin{aligned}
A Q+B \dot{Y}= & -Q\left[\xi\left(\frac{T_{2} D_{2} Y_{1}}{T_{1} D_{1} Y_{2}}-1\right)\left(Z_{1} \eta-\frac{T_{2} C_{v_{2}}}{\rho} C_{1} \eta\right)+\frac{T_{2}}{\alpha_{2} \rho_{2}} C_{1} \eta\right]+ \\
+ & \dot{Y}\left[-\xi\left(\frac{T_{2} D_{2} Y_{1}}{T_{1} D_{1} Y_{2}}-1\right)\left(\theta_{1}+\frac{T_{2} C_{v_{2}}}{\rho}\left(C_{2}-\frac{\rho}{\rho_{I}}\right)\right)+\frac{T_{2}}{\alpha_{2} \rho_{2}}\left(C_{2}-C_{1} \frac{\rho}{\rho_{I}}\right)\right], \\
A^{\prime} Q+B^{\prime} \dot{Y}= & Q\left[-\left(\frac{1}{\rho_{2}}-\frac{1}{\rho_{1}}\right) \frac{c_{1}^{2}}{\alpha_{1} \gamma_{1}} \rho_{1} \eta+\frac{S_{2} T_{2}}{\alpha_{2} \rho_{2}} C_{1} \eta-\xi \delta_{1}\left(Z_{1} \eta-\frac{T_{2} C_{v_{2}}}{\rho} C_{1} \eta\right)\right] \\
+ & \dot{Y}\left\{\left(\frac{1}{\rho_{2}}-\frac{1}{\rho_{1}}\right) \frac{c_{1}^{2}}{\alpha_{1} \gamma_{1}}\left(\rho-\rho_{1} \frac{\rho}{\rho_{I}}\right)-\frac{S_{2} T_{2}}{\alpha_{2} \rho_{2}}\left(C_{2}-C_{1} \frac{\rho}{\rho_{I}}\right)\right. \\
& \left.\quad-\xi \delta_{1}\left[\theta_{1}+\frac{T_{2} C_{v_{2}}}{\rho}\left(C_{2}-\frac{\rho}{\rho_{I}}\right)\right]\right\},
\end{aligned}
$$

where

$$
\delta_{1}=\left(\frac{1}{\rho_{2}}-\frac{1}{\rho_{1}}\right) \frac{c_{1}^{2} \rho_{1}}{T_{1} \gamma_{1}}+S_{1}-S_{2} \frac{T_{2} D_{2} Y_{1}}{T_{1} D 1 Y_{2}} .
$$

Considering the system (25), we can define the coefficients $A, B, A^{\prime}, B^{\prime}$ as follows:

$$
\begin{aligned}
& A=-\xi\left[\left(\frac{T_{2} D_{2} Y_{1}}{T_{1} D_{1} Y_{2}}-1\right)\left(Z_{1} \eta-\frac{T_{2} C_{v_{2}}}{\rho} C_{1} \eta\right)+\frac{T_{2}}{\alpha_{2} \rho_{2}} C_{1} \eta\right] \\
& B=-\xi\left(\frac{T_{2} D_{2} Y_{1}}{T_{1} D_{1} Y_{2}}-1\right)\left[\theta_{1}+\frac{T_{2} C v_{2}}{\rho}\left(C_{2}-\frac{\rho}{\rho_{I}}\right)\right]+\frac{T_{2}}{\alpha_{2} \rho_{2}}\left(C_{2}-C_{1} \frac{\rho}{\rho_{I}}\right) \\
& A^{\prime}=-\left(\frac{1}{\rho_{2}}-\frac{1}{\rho_{1}}\right) \frac{c_{1}^{2}}{\alpha_{1} \gamma_{1}} \rho_{1} \eta+\frac{S_{2} T_{2}}{\alpha_{2} \rho_{2}} C_{1} \eta-\xi \delta_{1}\left(Z_{1} \eta-\frac{T_{2} C_{v_{2}}}{\rho} C_{1} \eta\right) \\
& B^{\prime}=\left(\frac{1}{\rho_{2}}-\frac{1}{\rho_{1}}\right) \frac{c_{1}^{2}}{\alpha_{1} \gamma_{1}}\left(\rho-\rho_{1} \frac{\rho}{\rho_{I}}\right)-\frac{S_{2} T_{2}}{\alpha_{2} \rho_{2}}\left(C_{2}-C_{1} \frac{\rho}{\rho_{I}}\right)+ \\
&-\xi \delta_{1}\left[\theta_{1}+\frac{T_{2} C_{v_{2}}}{\rho}\left(C_{2}-\frac{\rho}{\rho_{I}}\right)\right]
\end{aligned}
$$

\subsection{Step 3: Mass fraction and density positivity}

The approximation of heat and mass transfer terms allow the calculation of source terms of volume fraction and mass conservative equations, but there is no guarantee that positivity of the solution be preserved. Assuming an evaporation process for example, the mass source terms estimated can be larger than the liquid mass fraction that can evaporate. So, as in [19, a limitation is placed on the source terms, by determining the maximum admissible values, as follows:

$$
S_{\max , \alpha_{1}}=\left\{\begin{array}{ll}
\frac{1-\alpha_{1}}{\Delta t} & \text { if } S_{\max }>0 \\
\frac{-\alpha_{1}}{\Delta t} & \text { otherwise, }
\end{array} \quad S_{\max , Y_{1}}= \begin{cases}\frac{\left(1-\alpha_{1}\right) \rho_{2}}{\Delta t} & \text { if } S_{\max }>0 \\
\frac{-\alpha_{1} \rho_{1}}{\Delta t} & \text { otherwise. }\end{cases}\right.
$$


Thus, if $\left|S_{\max , \alpha_{1}}\right|>\left|S_{\alpha_{1}}\right|$ and $\left|S_{\max , Y_{1}}\right|>\left|S_{Y_{1}}\right|$, the source terms is used.

Otherwise, if the limit value is not respected, a new method is introduced in order to avoid an integration of the system over a fractional hydrodynamic time step, as it is done in [19, 20]. We assumed that the mixture is composed nearly of the species $k$ that has the highest entropy, using an idea similar to [21, 33. In particular, let us indicate with $\star$ the variable value at the end of positivity system check control:

- if $s_{g}^{\star}>s_{l}^{\star}$, then the vapor volume fraction, $\alpha^{\star \star}$, is fixed to $1-\epsilon_{1}$,

- otherwise, if $s_{g}^{\star}<s_{l}^{\star}$, then $\alpha^{\star \star}$ is fixed to $\epsilon_{1}$,

where, as explained in Section 3.2, $\epsilon_{1}=10^{-8}$.

Knowing $\alpha^{\star \star}$, it can be used in the following system in order to find the new equilibrium pressure, $P^{\star \star}$, and temperature, $T^{\star \star}$, and, then the other thermodynamic variables (a stiffened equation of state is assumed) :

$$
\begin{aligned}
\rho^{\star \star} & =\left(\alpha_{1} \rho_{1}\right)^{\star \star}+\left(\alpha_{2} \rho_{2}\right)^{\star \star}=\rho^{\star} \\
(\rho e)^{\star \star} & =\left(\alpha_{1} \rho_{1} e_{1}\right)^{\star \star}+\left(\alpha_{2} \rho_{2} e_{2}\right)^{\star \star}=(\rho e)^{\star} \\
\rho_{k}^{\star \star}\left(P^{\star \star}, T^{\star \star}\right) & =\frac{P^{\star \star}+P_{\infty, k}}{C v_{k} \Gamma_{k} T^{\star \star}} \\
(\rho e)_{k}^{\star \star}\left(P^{\star \star}, T^{\star \star}\right) & =\frac{P^{\star \star}+P_{\infty, k} \gamma_{k}}{\Gamma_{k}}+\rho_{k}^{\star \star} q
\end{aligned}
$$

where $\Gamma=\gamma-1$. By replacing the phase density, $\rho_{k}^{\star \star}$, and the phase energy $(\rho e)_{k}^{\star \star}$, in the first two equations of system (27), we obtain a single quadratic equation for $P^{\star \star}$ :

$$
\begin{aligned}
T^{\star \star} & =\frac{\alpha_{1}^{\star \star} C v_{2} \Gamma_{2}\left(P^{\star \star}+P_{\infty, 1}\right)+\alpha_{2}^{\star \star} C v_{1} \Gamma_{1}\left(P^{\star \star}+P_{\infty, 2}\right)}{\rho^{\star} C v_{1} \Gamma_{1} C v_{2} \Gamma_{2}} \\
0 & =\left(P^{\star \star}\right)^{2}+b P^{\star \star}+d
\end{aligned}
$$

and:

$b=\frac{F_{1}}{F_{2}}+\frac{q_{1}\left(\alpha_{1}^{\star \star} C_{v_{2}} \Gamma_{2}\right)+q_{2}\left(\alpha_{2}^{\star \star} C_{v_{1}} \Gamma_{1}\right)}{Z_{2}}+F_{2} \frac{P_{\infty, 1}\left(\alpha_{1}^{\star \star} C_{v_{2}} \Gamma_{2}\right)+P_{\infty, 2}\left(\alpha_{2}^{\star \star} C_{v_{1}} \Gamma_{1}\right)}{\rho^{\star} \Gamma_{1} \Gamma_{2}}$

$d=\frac{F_{1}}{Z_{2}} \frac{P_{\infty, 1}\left(\alpha_{1}^{\star \star} C_{v_{2}} \Gamma_{2}\right)+P_{\infty, 2}\left(\alpha_{2}^{\star \star} C_{v_{1}} \Gamma_{1}\right)}{\rho^{\star} \Gamma_{1} \Gamma_{2}}+\frac{P_{\infty, 1} q_{1}\left(\alpha_{1}^{\star \star} C_{v_{2}} \Gamma_{2}\right)+P_{\infty, 2} q_{2}\left(\alpha_{2}^{\star \star} C_{v_{1}} \Gamma_{1}\right)}{Z 2}$

where

$$
\begin{gathered}
F_{1}=P_{\infty, 1} \gamma_{1} \frac{\left(\alpha_{1}^{\star \star} C_{v_{2}} \Gamma_{2}\right)}{C_{v_{2}}}+P_{\infty, 2} \gamma_{2} \frac{\left(\alpha_{2}^{\star \star} C_{v_{1}} \Gamma_{1}\right)}{C_{v_{1}}}-(\rho e)^{\star} \Gamma_{1} \Gamma_{2} \\
F_{2}=\alpha_{1}^{\star \star} \Gamma_{2}+\alpha_{2}^{\star \star} \Gamma_{1} \\
Z_{2}=F_{2} \frac{\left(\alpha_{1}^{\star \star} C_{v_{2}} \Gamma_{2}\right)+\left(\alpha_{2}^{\star \star} C_{v_{1}} \Gamma_{1}\right)}{\rho^{\star \star} \Gamma_{1} \Gamma_{2}} .
\end{gathered}
$$

Then, by solving the single quadratic equation of $P^{\star \star}$, we select the physically admissible solution of the quadratic equation that maximizes the total entropy $s^{\star \star}=Y_{1}^{\star \star} s_{1}^{\star \star}+Y_{2}^{\star \star} s_{2}^{\star \star}$, where $Y_{k}^{\star \star}=\left(\alpha_{k}^{\star \star} \rho_{k}^{\star \star}\right) / \rho^{\star \star}$

This procedure allows to reduce the computational cost compared to [19, 20]: it is not necessary to integrate over a fractional hydrodynamic time step. 


\section{Thermodynamic closure}

As we have previously mentioned, we deal with pure fluid and artificial mixture zone, thus the EOS must be able to describe flows both in pure fluids and mixture zones.

In order to clarify this point, let us remember that, in the system (3), the last two equations are the momentum and energy conservation equations for the mixture, in which, a mixture pressure $\mathrm{P}$ appears. For this reason, a mixture EOS for extrapolating the value of $\mathrm{P}$, is required.

\subsection{Stiffened Gas EOS for pure fluid}

The Stiffened Gas EOS is usually used for shock dynamics and its robustness for simulating two-phase flow with or without mass transfer has been amply demonstrated (see for example [29, 15, 34, 26, 35]). It can be written as follows:

$$
\begin{gathered}
P(\rho, e)=(\gamma-1)(e-q) \rho-\gamma P_{\infty}, \\
e(\rho, T)=T c_{v}+\frac{P_{\infty}}{\rho}+q \\
\rho(P, T)=\frac{P+P_{\infty}}{(\gamma-1) c_{v} T} \\
h(T)=\gamma c_{v} T \\
s(P, T)=c_{v} \ln \frac{T^{\gamma}}{\left(P+P_{\infty}\right)^{(\gamma-1)}}+q^{\prime}, \\
g(P, T)=h(T)-T s(P, T),
\end{gathered}
$$

where $P, \rho$ and $e$ are the pressure, the density and the energy, respectively. The polytropic coefficient $\gamma$ is the constant ratio of specific heat capacities $\gamma=c_{p} / c_{v}$, $P_{\infty}$ is a constant reference pressure and $q$ is the energy of the fluid at a given reference state. Moreover, $T, c_{v}$ and $h$ are the temperature, the specific heat at constant volume and the enthalpy, respectively. The speed of sound, defined as $c^{2}=\left(\frac{\partial P}{\partial \rho}\right)_{s}$ can be computed as follows:

$$
c^{2}=\gamma \frac{P+P_{\infty}}{\rho}=(\gamma-1) c_{p} T
$$

where $c^{2}$ remains strictly positive (for $\gamma>1$ ). It ensures the hyperbolicity of the system and the existence of a convex mathematical entropy [36.

\subsection{SG EOS based mixture}

The EOS for the mixture can be easily obtained using the EOS of the single phase. The aim is now to obtain the mixture pressure. The starting point is the mixture energy equation:

$$
\rho e=\alpha_{1} \rho_{1} e_{1}+\alpha_{2} \rho_{2} e_{2} .
$$


The energy of each phase, $e_{k}=\frac{P+P_{\infty} \gamma}{\rho_{k}\left(\gamma_{k}-1\right)}$, can be replaced obtaining the mixture total energy as a function of the phase pressures. Under pressure equilibrium, the following expression for the pressure mixture can be obtained:

$$
P\left(\rho, e, \alpha_{k}\right)=\frac{\rho\left(e-\frac{\alpha_{1} \rho_{1} q_{1}}{\rho}-\frac{\alpha_{2} \rho_{2} q_{2}}{\rho}\right)-\left(\frac{\alpha_{1} \gamma_{1} P_{\infty, 1}}{\gamma_{1}-1}+\frac{\alpha_{2} \gamma_{2} P_{\infty, 2}}{\gamma_{2}-1}\right)}{\frac{\alpha_{1}}{\gamma_{1}-1}+\frac{\alpha_{2}}{\gamma_{2}-1}}
$$

\subsection{Definition of $\chi_{k}$ and $\kappa_{k}$}

For the SG EOS, it is trivial to compute the coefficients of the semi-discrete equation (5) as follows:

$$
\begin{gathered}
\chi_{k}=\left(\frac{\partial e_{k}}{\partial P_{k}}\right)_{\rho_{k}}=\frac{1}{(\gamma-1) \rho}, \\
\kappa_{k}=\left(\frac{\partial e_{k}}{\partial \rho_{k}}\right)_{P_{k}}=-\frac{P+\gamma P_{\infty}}{(\gamma-1) \rho^{2}} .
\end{gathered}
$$

\section{$5 \quad$ Extension to six and seven-equation model}

In this section, the cavitation model is extended to more complex systems of equations. Some hypothesis used in the previous sections are no more valid. In particular, the attention is focused on six (single velocity) and seven-equation models, respectively. Note that step 1 consists always in finding the solution for the hyperbolic part of the model, i.e. without the heat and mass transfer terms. As a consequence, a different model is associated to a different discretization of the hyperbolic part. We see that step 1 remains unchanged, contrarily to steps 2 and 3.

\subsection{Modeling cavitation with the seven-equation model}

For clearly explaining the natural extension of the model proposed in the previous section to the seven-equation model, let us define the different steps, as follows.

\subsubsection{Step 1 for a seven-equation model}

Let us suppose here that each phase has its own pressure and velocity (see see system (11)). However, during the mass transfer, the two phases have the same pressure, that is equal to the saturation pressure. So, a relaxation procedure should be applied in order to obtain a single pressure system as in [27, 21].

We have assumed that the pressure relaxation is faster than the temperature and the Gibbs free energy relaxation: Step 1 is necessary to find the solution of the system (11) without the heat and mass transfer terms and to apply a pressure relaxation procedure. In this way, variables summarized in Table 4 are obtained. 


\begin{tabular}{c|c}
\hline \multicolumn{2}{c|}{ Step 1 for a seven-equation model } \\
\hline \hline IN & OUT \\
\hline$\alpha_{1}^{0}\left(\alpha_{2}^{0}=1-\alpha_{1}^{0}\right)$ & $\alpha_{1}^{\star}\left(\alpha_{2}^{\star}=1-\alpha_{1}^{\star}\right)$ \\
$\rho_{1}^{0}$ and $\rho_{2}^{0}$ & $\rho_{1}^{\star}$ and $\rho_{2}^{\star}$ \\
$v_{1}^{0}$ and $v_{2}^{0}$ & $v_{1}^{\star}$ and $v_{2}^{\star}$ \\
$P_{1}^{0}$ and $P_{2}^{0}$ & $P^{\star}$ \\
$e_{1}^{0}$ and $e_{2}^{0}$ & $e_{1}^{\star}$ and $e_{2}^{\star}$ \\
$T_{1}^{0}$ and $T_{2}^{0}$ & $T_{1}^{\star}$ and $T_{2}^{\star}$ \\
$g_{1}^{0}$ and $g_{2}^{0}$ & $g_{1}^{\star}$ and $g_{2}^{\star}$ \\
\hline
\end{tabular}

Table 4: Variables used in Step 1 for a seven-equation model

\subsubsection{Step 2 for a seven-equation model}

As in the five-equation model, if the system is in metastable conditions, i.e. $\left(T_{k}>T_{\text {sat }}\right)$ and at the interface $\epsilon_{1}<\alpha_{1}<1-\epsilon_{1}$, the ODE system for the seven-equation model (see system (10) is as follows:

$$
\begin{aligned}
& \frac{\partial \alpha_{1}}{\partial t}=\frac{\alpha_{1} \alpha_{2}}{\alpha_{2} \rho_{1} c_{1}^{2}+\alpha_{1} \rho_{2} c_{2}^{2}}\left(\frac{\Gamma_{1}}{\alpha_{1}}+\frac{\Gamma_{2}}{\alpha_{2}}\right) Q+\frac{\rho}{\rho_{I}} \dot{Y} \\
& \frac{\partial\left(\alpha_{1} \rho_{1}\right)}{\partial t}=\rho \dot{Y} \\
& \frac{\partial\left(\alpha_{1} \rho_{1} v_{1}\right)}{\partial t}=v_{I} \rho \dot{Y} \\
& \frac{\partial\left(\alpha_{1} \rho_{1} E_{1}\right)}{\partial t}=Q+E_{I} \rho \dot{Y} \\
& \frac{\partial\left(\alpha_{2} \rho_{2}\right)}{\partial t}=-\rho \dot{Y} \\
& \frac{\partial\left(\alpha_{2} \rho_{2} v_{2}\right)}{\partial t}=-v_{I} \rho \dot{Y} \\
& \frac{\partial\left(\alpha_{2} \rho_{2} E_{2}\right)}{\partial t}=-Q-E_{I} \rho \dot{Y} .
\end{aligned}
$$

Several definitions of the interface pressure, $P_{I}$, and velocity, $v_{I}$, exist in the literature [29, 37]. For example, in [12, $v_{I}$ is assumed equal to liquid velocity, $v_{2}$, and $P_{I}$ equal to vapor pressure, $P_{1}$. Otherwise, more complex definitions can be used, as in 38]:

$$
\left\{\begin{array}{c}
P_{I}=\frac{Z_{1} P_{2}+Z_{2} P_{1}}{Z_{1}+Z_{2}}+\operatorname{sign}\left(\frac{\partial \alpha_{1}}{\partial x}\right) \frac{\left(u_{2}-u_{1}\right)}{Z_{1}+Z_{2}} \\
u_{I}=\frac{Z_{1} u_{1}+Z_{2} u_{2}}{Z_{1}+Z_{2}}+\operatorname{sign}\left(\frac{\partial \alpha_{1}}{\partial x}\right) \frac{P_{2}+P_{1}}{Z_{1}+Z_{2}}
\end{array}\right.
$$

where $Z=\rho c$ represents the acoustic impedance and $c$ is the speed of sound.

The variable $\rho_{I}$ is the interface density and it is defined by means of the equation (10). Remembering that $\alpha_{2}=1-\alpha_{1}$, the unknowns of the system (135) are $\alpha_{1}, \rho_{k}, v_{k}, P, e_{k}, Q, \dot{Y}, E_{I}$. Then, to close the system, the equation of state for defining the energy as function of pressure and density is used for both phases. The interface energy, $E_{I}$, will be developed in section 5.1.4. Moreover, 
a thermo-chemical relaxation for finding $Q$ and $\dot{Y}$ is imposed, as follows:

$$
\begin{aligned}
& \frac{\partial \Delta T}{\partial t}=A Q+B \dot{Y} \\
& \frac{\partial \Delta g}{\partial t}=A^{\prime} Q+B^{\prime} \dot{Y}
\end{aligned}
$$

The procedure described in Section 3.2 .2 can be applied in this case without problem, i.e. the mass conservation equations and the mixture internal energy ones can be used in order to find the value of the four coefficients $A, B, A^{\prime}$ and $B^{\prime}$. However, the only mixture variable that remains constant from the step 1 to the step 2 is $\rho$ (see Table (5) ). This means that, as in Section 3.2.2, the development of equations (15) and (17) do not change and equations (16) and (18) can be obtained. Note also that Eq. (19) is not valid anymore. Then, remembering that, for a seven-equation model,

$$
\alpha_{1} \rho_{1} \frac{\partial u_{1}}{\partial t}=\rho \dot{Y}\left(u_{I}-u_{1}\right)
$$

and

$$
\alpha_{2} \rho_{2} \frac{\partial u_{2}}{\partial t}=\rho \dot{Y}\left(u_{I}-u_{2}\right)
$$

the mixture energy equation can be written as follows:

$$
\begin{gathered}
\frac{\partial \rho e}{\partial t}=\frac{\partial\left(\alpha_{1} \rho_{1} E_{1}+\alpha_{2} \rho_{2} E_{2}\right)}{\partial t}-\frac{\partial\left[\alpha_{1} \rho_{1}\left(\frac{1}{2} u_{1}^{2}\right)+\alpha_{2} \rho_{2}\left(\frac{1}{2} u_{1}^{2}\right)\right]}{\partial t}= \\
=-\frac{1}{2} \rho \dot{Y}\left(u_{2}^{2}-2 u_{2} u_{I}+2 u_{1} u_{I}-u_{1}^{2}\right)=-\beta \dot{Y}
\end{gathered}
$$

By coupling the equation (20) and its developments (until the equation (22) ) with the equation (40), this last one can be written as follows:

$$
\frac{\partial \rho e}{\partial t}=\theta 1 \dot{Y}+Z_{1} \eta Q+Y_{1} C_{v_{1}} \frac{\partial T_{1}}{\partial t}+Y_{2} C_{v_{2}} \frac{\partial T_{2}}{\partial t}=-\beta \dot{Y} .
$$

As a consequence, by coupling equations (41), (16) and (18), the following new values for the coefficients $A, B, A^{\prime}$ and $B^{\prime}$ for the seven-equation model can be obtained:

$$
\begin{aligned}
A & =-\left[\xi\left(\frac{T_{2} D_{2} Y_{1}}{T_{1} D_{1} Y_{2}}-1\right)\left(Z_{1} \eta-\frac{T_{2} C_{v_{2}}}{\rho} C_{1} \eta\right)+\frac{T_{2}}{\alpha_{2} \rho_{2}} C_{1} \eta\right] \\
B & =-\xi\left(\frac{T_{2} D_{2} Y_{1}}{T_{1} D_{1} Y_{2}}-1\right)\left[\theta_{1}+\frac{T_{2} C_{v_{2}}}{\rho}\left(C_{2}-\frac{\rho}{\rho_{I}}+\beta\right)\right]+\frac{T_{2}}{\alpha_{2} \rho_{2}}\left(C_{2}-C_{1} \frac{\rho}{\rho_{I}}\right) \\
A^{\prime} & =-\left(\frac{1}{\rho_{2}}-\frac{1}{\rho_{1}}\right) \frac{c_{1}^{2}}{\alpha_{1} \gamma_{1}} \rho_{1} \eta+\frac{S_{2} T_{2}}{\alpha_{2} \rho_{2}} C_{1} \eta-\xi \delta_{1}\left(Z_{1} \eta-\frac{T_{2} C v_{2}}{\rho} C_{1} \eta\right) \\
B^{\prime} & =\left(\frac{1}{\rho_{2}}-\frac{1}{\rho_{1}}\right) \frac{c_{1}^{2}}{\alpha_{1} \gamma_{1}}\left(\rho-\rho_{1} \frac{\rho}{\rho_{I}}\right)-\frac{S_{2} T_{2}}{\alpha_{2} \rho_{2}}\left(C_{2}-C_{1} \frac{\rho}{\rho_{I}}\right)+ \\
-\xi \delta_{1} & {\left[\theta_{1}+\frac{T_{2} C_{v_{2}}}{\rho}\left(C_{2}-\frac{\rho}{\rho_{I}}+\beta\right)\right] . }
\end{aligned}
$$

Remark that coefficients $A$ and $A^{\prime}$ are equal to the coefficients of Section 3.2 .2 . Instead, coefficients $B$ and $B^{\prime}$ are different only for the term $\beta$. 
Step 2 for the seven-equation model

\begin{tabular}{c|c|c}
\multicolumn{2}{c}{ (Only on the interface, if $\epsilon_{1}<\alpha_{1}<1-\epsilon_{1}$ and $\left.T_{k}>T_{\text {sat }}\left(P^{\star}\right)\right)$} \\
\hline \hline IN & OUT & Constant mixture variables \\
\hline$\alpha_{1}^{\star}\left(\alpha_{2}^{\star}=1-\alpha_{1}^{\star}\right)$ & $\alpha_{1}^{\star \star}\left(\alpha_{2}^{\star \star}=1-\alpha_{1}^{\star \star}\right)$ & \\
$\rho_{1}^{\star}$ and $\rho_{2}^{\star}$ & $\rho_{1}^{\star \star}$ and $\rho_{2}^{\star \star}$ & \\
$v^{\star}$ & $v_{1}^{\star \star}$ and $v_{2}^{\star \star}$ & $\rho^{\star \star}=\rho^{\star}$ \\
$P^{\star}$ & $P^{\star \star}$ & \\
$e_{1}^{\star}$ and $e_{2}^{\star}$ & $e_{1}^{\star \star}$ and $e_{2}^{\star \star}$ & $T^{\star \star}$ \\
$T_{1}^{\star}$ and $T_{2}^{\star}$ & $g^{\star \star}$ & \\
$g_{1}^{\star}$ and $g_{2}^{\star}$ &
\end{tabular}

Table 5: Variables used in Step 2 for a seven-equation model. $\rho=\left(\alpha_{1} \rho_{1}\right)+$ $\left(\alpha_{2} \rho_{2}\right) ; \rho e=\left(\alpha_{1} \rho_{1} e_{1}\right)+\left(\alpha_{2} \rho_{2} e_{2}\right) ; E=e+\frac{1}{2} v^{2}$.

\subsubsection{Step 3 for a seven-equation model}

For the seven-equation model, Step 3 is equal to the one described in Section 3.3 without other modifications.

\subsubsection{Determination of $E_{I}$}

Let us now focus the attention on the definition of the variable $E_{I}$ (see system of equations 35). Let us start the demonstration from the gas total energy relation:

$$
\frac{\partial\left(\alpha_{1} \rho_{1} E_{1}\right)}{\partial t}=\alpha_{1} \rho_{1} \frac{\partial\left(e_{1}+\frac{1}{2} u_{1}^{2}\right)}{\partial t}+E_{1} \rho \dot{Y}=Q+E_{I} \rho \dot{Y} .
$$

By substituting equation (38) in the equation (42), this last one becomes:

$$
\alpha_{1} \rho_{1} \frac{\partial e_{1}}{\partial t}+u_{1}\left(u_{I}-u_{1}\right) \rho \dot{Y}+E_{1} \rho \dot{Y}=Q+E_{I} \rho \dot{Y}
$$

The energy can be written as a function of density and temperature, $e_{1}\left(T_{1}, \rho_{1}\right)$, so the temporal derivative can be written as follows:

$$
\begin{aligned}
&\left.\frac{\partial e_{1}}{\partial t}\left(T_{1}, \rho_{1}\right)\right)=\left.\frac{\partial e_{1}}{\partial T_{1}}\right|_{\rho_{1}} \frac{\partial T_{1}}{\partial t}+\left.\frac{\partial e_{1}}{\partial \rho_{1}}\right|_{T_{1}} \frac{\partial \rho_{1}}{\partial t}= \\
&=c_{v, 1} \xi\left\{-\dot{Y}\left[\theta_{1}+\frac{T_{2} C v_{2}}{\rho}\left(C_{2}-\frac{\rho}{\rho_{I}}+\beta\right)\right]+\right. \\
&\left.\quad-Q\left(Z 1 \eta-\frac{T_{2} C v_{2}}{\rho} C 1 \eta\right)\right\} .
\end{aligned}
$$

Then, by replacing the equation (45) into the equation (43), after some manipulations, the interface energy can be defined as follows:

$$
\begin{aligned}
E_{I}=-\alpha_{1} \rho_{1} c_{v, 1} & \frac{\xi}{\rho}\left[\theta_{1}+\frac{T_{2} C v_{2}}{\rho}\left(C_{2}-\frac{\rho}{\rho_{I}}+\beta\right)\right]+ \\
+ & P_{\infty, 1}\left(\frac{1}{\rho_{I}}-\frac{1}{\rho_{1}}\right)\left(u_{1} u_{I}-\frac{1}{2} u_{1}^{2}+e_{1}\right)+ \\
& +\frac{Q}{\rho \dot{Y}}\left[P_{\infty, 1} \eta-\alpha_{1} \rho_{1} c_{v, 1} \xi\left(Z 1 \eta-\frac{T_{2} C v_{2}}{\rho} C 1 \eta\right)-1\right] .
\end{aligned}
$$




\subsection{Modeling cavitation for a six-equation model}

The six-equation model is based on the assumption that the phase velocities are equal. This can be represented by the following system of equations:

$$
\begin{aligned}
\frac{\partial \alpha_{1}}{\partial t}+v_{I} \frac{\partial \alpha_{1}}{\partial x} & =\mu\left(P_{1}-P_{2}\right)+\eta Q+\frac{\rho}{\rho_{I}} \dot{Y} \\
\frac{\partial\left(\alpha_{1} \rho_{1}\right)}{\partial t}+\frac{\partial\left(\alpha_{1} \rho_{1} v_{1}\right)}{\partial x}= & \rho \dot{Y} \\
\frac{\partial\left(\alpha_{2} \rho_{2}\right)}{\partial t}+\frac{\partial\left(\alpha_{2} \rho_{2} v_{2}\right)}{\partial x}= & -\rho \dot{Y} \\
\frac{\partial(\rho v)}{\partial t}+\frac{\partial\left(\rho v^{2}+\alpha_{1} P_{1}+\alpha_{2} P_{2}\right)}{\partial x}= & 0 \\
\frac{\partial\left(\alpha_{1} \rho_{1} E_{1}\right)}{\partial t}+\frac{\partial\left(\alpha_{1}\left(\rho_{1} E_{1}+P_{1}\right) v_{1}\right)}{\partial x}= & P_{I} v_{I} \frac{\partial \alpha_{1}}{\partial x}+\lambda v_{I}\left(v_{2}-v_{1}\right)+ \\
& +\mu P_{I}\left(P_{1}-P_{2}\right)+Q+E_{I} \rho \dot{Y} \\
\frac{\partial\left(\alpha_{2} \rho_{2} E_{2}\right)}{\partial t}+\frac{\partial\left(\alpha_{2}\left(\rho_{2} E_{2}+P_{2}\right) v_{2}\right)}{\partial x}= & P_{I} v_{I} \frac{\partial \alpha_{2}}{\partial x}-\lambda v_{I}\left(v_{2}-v_{1}\right)+ \\
& -\mu P_{I}\left(P_{1}-P_{2}\right)-Q-E_{I} \rho \dot{Y} .
\end{aligned}
$$

In this case also, it is assumed that, during the mass transfer, the two phases have the same pressure, equal to the saturation pressure. So, a relaxation procedure should be applied in order to obtain a single pressure system as in [21, 20]. This means, that, close to the interface, it is equivalent to a five equation model. As a consequence, all the considerations developed in Section 3.2. and coefficients $A, B, A^{\prime}$ and $B^{\prime}$ found in Section 3.2.2, can be equally used for a six-equation model.

\section{$6 \quad$ Results}

All the numerical simulations presented in this paper are performed by means of a five equation model coupled with the cavitation model of section 3.2 .2 Several numerical test-case are performed. Then, the robustness of the numerical method is assessed on some of the most used numerical configuration in the literature for estimating efficiency of two-phase compressible models. In particular, the aim is to i) show the influence of heat and mass transfer, ii) validate the results by comparison with respect to the experimental data and to the existing state-of-art in terms of numerical simulation, iii) estimate the prediction in terms of important quantities from a physical point of view.

All the numerical test-cases are performed by using liquid and vapor of dodecane or of water. Physical parameters for each test-case are summarized in Table 6. A second order has been performed for all the numercial test-cases by means a Muscl scheme (see 29] for more details), coupled with a Relaxation solver (31, 26]) and a Minmod limiter. Note also that a mesh-convergence study has been done for each test-case, even if it is not reported here for brevity. The first test case is a classical two phase shock-tube that allows to observe the appearance of the evaporation wave and is a classical test-case in literature. Secondly, the experiment of Simoes-Moreira 39] is reproduced. It reproduces an experimental two phase shock-tube that allows to validate the cavitation model 
with respect to the experimental data. The last two test cases are associated to a two-phase expansion tube with different initial phase velocities, which is widely used in literature 21. These test cases allow to observe the appearance of the evaporation wave in a mixture and not in a pure liquid as in the case of a shock-tube problem.

\begin{tabular}{|c|c|c|c|c|c|c|c|}
\hline \multirow{2}{*}{ TEST CASE } & \multirow{2}{*}{ FLUID } & \multicolumn{6}{|c|}{ SG EOS } \\
\hline & & $\gamma$ & $P_{\infty}[P a]$ & $C_{p}\left[\frac{J}{K g K}\right]$ & $C_{v}\left[\frac{J}{K g K}\right]$ & $\mathrm{q}$ & $q^{\prime}$ \\
\hline \multirow{2}{*}{$\begin{array}{c}1 \\
\text { Shock tube }\end{array}$} & Liq. Dodecane & 2.35 & $4 \times 10^{8}$ & 2534 & 1077 & $-755 \times 10^{3}$ & 0 \\
\hline & Vap. Dodecane & 1.025 & 0 & 2005 & 1956 & $-237 \times 10^{3}$ & $-24.2 \times 10^{3}$ \\
\hline \multirow{3}{*}{$\begin{array}{c}2 \\
\text { Shock tube } \\
\text { experiments }\end{array}$} & & & & & & & \\
\hline & Liq. Dodecane & 2.35 & $4 \times 10^{8}$ & 2534 & 1077 & $-755 \times 10^{3}$ & 0 \\
\hline & Vap. Dodecane & 1.025 & 0 & 2005 & 1956 & $-237 \times 10^{3}$ & $-24.2 \times 10^{3}$ \\
\hline \multirow{2}{*}{$\begin{array}{c}3-4 \\
\text { Expansion tube }\end{array}$} & Liq. Water & 2.35 & $10^{9}$ & $\overline{4267}$ & $\overline{1816}$ & $-1167 \times 10^{3}$ & 0 \\
\hline & Vap. Water & 1.43 & 0 & 1487 & 1040 & $2030 \times 10^{3}$ & $-23 \times 10^{3}$ \\
\hline
\end{tabular}

Table 6: EOS coefficients for liquid and gas phases.

\subsection{TC1: Two-phase shock tube with mass transfer}

In this test-case, the shock tube is filled out with liquid dodecane on the left at high pressure $p_{l}=10^{8}$ and vapor dodecane on the right at atmospheric pressure $p_{g}=10^{5}$. Note that, for numerical reasons, each chamber contains a weak volume of the other fluid $\left(\alpha_{k}=10^{-8}\right)$. The diaphragm is located at $x=0.75 \mathrm{~m}$ (the tube is $1 \mathrm{~m}$ long) and the results are shown at a time of $t=473 \mu \mathrm{s}$. The computation are performed by using a 1000 mesh grid.

A comparison between the numerical solutions obtained with and without heat and mass transfer is performed for estimating the influence of these terms. Moreover, results are compared with the ones obtained by Pelanti and Shyue 21] (considering a pressure relaxation coupled with a pressure-thermo-chemical relaxation). These comparisons are reported in Fig. 4.

Looking at Fig. 4, the influence of heat and mass transfer can be observed. In fact, an additional evaporation wave appears between the rarefaction wave (at $0.05<x<0.12 m$ ) and the contact discontinuity (at $0.87<x<0.9 m$ ) (see Fig. 4k-d). The evaporation wave determines a strong increase of velocity (see Fig. 4 $\mathrm{b}$ ) and an increase of pressure at $0.12<x<0.8 \mathrm{~m}$ (see Fig. 4a). Note that this value of pressure corresponds to the saturation pressure.

From the comparison with Pelanti and Shyue [21, a very good agreement can be observed in terms of pressure and mixture density (Fig. 4a-c). The maximum value of velocity obtained by means of the present model is higher than the one obtained in [21] (nearly $7 \%$ of difference). Nevertheless, it does agree quantitatively with Saurel et al. solution given in [19] (Fig. 4b). In fact, in terms of vapor mass fraction, $Y_{v}$, the three models provide three different results (Fig. 41).

\subsection{TC2: Simoes-Moreira shock tube experiments}

This test case reproduce the experiments that have been carried out by SimoesMoreira and Shepherd 39. The shock tube is filled out with dodecane on the 
left at high pressure and vapor dodecane on the right at low pressure chamber $\left(p=10^{2} \mathrm{~Pa}\right.$ and $\left.10^{-4}\left[\mathrm{~kg} / \mathrm{m}^{3}\right]\right)$. The diaphragm is located at $x=0.75 \mathrm{~m}$ (the tube is $1 \mathrm{~m}$ long) and all computations were performed by using a 1000 mesh grid.

The velocity of an evaporation wave that propagates in metastable liquid, is measured for different initial temperatures of liquid dodecane. The pressures before and after the evaporation wave were measured in 39]. The same strategy proposed by Zein et al. 20] is followed here for identifying the pressure value on the left hand side of the shock tube. In particular, the initial pressure is fixed so that the pressure in the state before the evaporation front is equal to the measured value (see Table 7).

The front velocity is computed by measuring the space traced by the evaporation wave at different times. In particular, the evolution of starting point of the evaporation wave at $400 \mu s$ and $500 \mu s$ is observed for all computations.

These results are compared with the experimental data and the results obtained by Saurel et al. 19] and by Zein et al. 20, in Fig. 5. As for 20, these results are closer to the experimental points, by displaying a trend of increase of temperature. Moreover, a better agreement than Saurel et al. 19] can be observed. Nevertheless, an overestimation of the velocity front is obtained. This could be due to a strong influence of the variability of the initial conditions (that are affected by a non-negligible uncertainty) and to the coefficients of the EOS that could influence the computations. Nevertheless, remark that, with respect to the cavitation model proposed in Zein et al. [20], the present model has a lower computation cost as explained in Section 3.3. since it is not necessary to integrate over a fractional hydrodynamic time step. This aspects is highlighted in sections 6.3 and 6.4

Note also that experimental measurements are affected by an uncertainty, then, it is questionable which numerical solution is the most accurate.

\begin{tabular}{c|c|c|c|c}
\hline$T_{l}$ & $p_{l}$ & $\begin{array}{c}p_{\exp } \\
\text { (Before evaporation) }\end{array}$ & $\begin{array}{c}u_{E F}(\mathrm{~m} / \mathrm{s}) \\
\text { (Experimental) }\end{array}$ & $\begin{array}{c}u_{E F}(\mathrm{~m} / \mathrm{s}) \\
\text { (Computed) }\end{array}$ \\
\hline 453 & 1.5 & 0.24 & 0.253 & 0.23851 \\
473 & 2.2 & 0.33 & 0.309 & 0.37501 \\
489 & 3.0 & 0.44 & 0.390 & 0.42073 \\
503 & 3.9 & 0.59 & 0.472 & 0.55698 \\
523 & 5.0 & 0.83 & 0.648 & 0.75894 \\
543 & 7.5 & 1.19 & 0.837 & 0.9548 \\
563 & 11.0 & 1.91 & 1.381 & 1.6398 \\
573 & 13.0 & 2.12 & 1.578 & 1.8369 \\
\hline
\end{tabular}

Table 7: Initial liquid temperature on the left side, left liquid pressure, experimental pressure measured before the evaporation front, evaporation front velocity measured and computed front velocity. 


\subsection{TC3: two-phase expansion tube with mixture initial velocities $v= \pm 2 \mathrm{~m} / \mathrm{s}$}

Now, let us consider a tube of unit length, filled out with liquid water of density $\rho_{2}=1150 \mathrm{~kg} / \mathrm{m}^{3}$ at an atmospheric pressure $P=10^{5} \mathrm{~Pa}$. The liquid is assumed to contain a uniformly distributed small amount of vapor, $\alpha_{1}=10^{-2}$ in the whole domain. The liquid temperature is equal to $T_{2}=354.728 \mathrm{~K}$ and hence the vapor density $\rho_{1}=0.63 \mathrm{~kg} / \mathrm{m}^{3}$, by assuming the flow in thermal equilibrium $T_{2}=T_{1}$. The solution is shown at a time $\mathrm{t}=3.2 \mathrm{~ms}$ in Fig. 6. The computation is performed by using a mesh of 5000 cells. A velocity discontinuity is set at $\mathrm{x}=0.5 \mathrm{~m}$ at initial time. We set $v=-2 \mathrm{~m} / \mathrm{s}$ on the left and $v=2 \mathrm{~m} / \mathrm{s}$ on the right of the discontinuity.

As it can be observed in Fig. [6-d, the vapor volume fraction increase is located in the center of the tube, where a mechanical expansion exists. It corresponds to a region, where the pressure attain the saturation value of about $0.5 \mathrm{~Pa}$ (see Fig. 6a). Also in this test case, the comparison with the results obtained by Pelanti and Shyue 21] and by Zein et al. 20, shows a very good agreement in terms of pressure and velocity (Fig. 6a-b). Some differences can be observed in terms of vapor volume fraction and vapor mass fraction, $Y_{v}$, (see Fig. 6k-d), for which the present model provides a maximum value that is between those ones obtained by the other two cavitation models.

Nevertheless, as in [21], our results are obtained with a reduction of the computational cost with respect to Zein et al. 20. In fact, in the case of heat and mass transfer, the CPU time is reduced of nearly 9 times with respect to the method proposed in 20] (with respect to our implementation of the method). In fact, the CPU time is of $2.15 \mathrm{~h}\left(32000\right.$ time steps and $\left.\mathrm{dt}=1.0 * 10^{-7}\right)$ and of $18.838 \mathrm{~h}$ (763550 time steps) for our computation and for [20, respectively. Note that computational cost description in [20 makes not possible a more accurate comparison.

\subsection{TC4: two-phase expansion tube with mixture initial velocities $v= \pm 500 \mathrm{~m} / \mathrm{s}$}

In this section, the analogous test of Section 6.3 but with different initial conditions is presented. In particular, the rarefaction effects are increased by setting the initial velocity $v=-500 \mathrm{~m} / \mathrm{s}$ on the left and $v=500 \mathrm{~m} / \mathrm{s}$ on the right. The computation is performed by using a mesh of 5000 cells and the solution is shown at a time $\mathrm{t}=0.58 \mathrm{~ms}$ (see Fig. 6).

Since the initial velocity is very large (Fig. 6b), the evaporation is consequently much more intense in the center of the tube, where the vapor volume fraction attains the value of 1 (see Fig. 6r).

The comparison with the results obtained by Pelanti and Shyue 21] and Zein et al. 20] shows a very good agreement in terms of pressure, velocity and vapor volume fraction (Fig. 6a-c). As in the TC3 (see Section 6.3), some differences can be observed in terms of vapor mass fraction, $Y_{v}$, (see Fig. 6 $\mathrm{d}$ ). In particular, a difference of nearly $20 \%$ can be observed for the maximum. Since no experimental data are available for this test-case, it should be useful to perform a sensitivity analysis by characterizing the amount of uncertainty to consider for the initial conditions.

Also in this test case, a reduction of the computational cost is observed with 
respect to Zein et al. [20]. In fact, when considering heat and mass transfer, the CPU time is reduced of nearly 2.5 times with respect to [20]. In particular, the CPU time is of $1.45 \mathrm{~h}$ (5800 time steps and $\mathrm{dt}=1.0 * 10^{-7}$ ) and of 3.372 h (186601 time steps) for our computation and for [20], respectively. Note that computational cost description in 20] makes not possible a more accurate comparison.

\section{Conclusions and perspective}

The present work is devoted to the development of a new cavitation model in order to reproduce compressible two-phase flow with mass transfer. In particular, starting from the model developed in [19] for a five equation model, the present model permits to reduce the computational cost by coupling the treatment of heat and mass transfer terms proposed in [19] for a five-equation model, with the solution admissibility procedure of [21. This allows to reduce the computational cost by preserving, always, the positivity of solution.

Moreover, the model has been theoretically extended to more complex models, such as the six and seven-equation models, showing the flexibility of the present cavitation model, a good accuracy and a reduced computational cost.

Several results have been presented for highlighting the main contributions of the proposed approach. Well-known numerical configurations used in the literature for assessing robustness of compressible two-phase models have been considered. The proposed method yields very good results in terms of accuracy with respect to the experimental data and numerical solutions known in literature. In some results, the effect of the heat and mass transfer have been assessed and evaluated. In particular, shock tube and two-phase expansion tube problems have been computed. The appearance of the evaporation wave has been observed in the case of pure liquid phase and not-pure phase. The comparison with experimental data and other numerical methods known in the literature [21, 19, 20] showed a very good agreement, thus assessing the proposed model.

Note that for some quantities of interest, slight differences with respect to other numerical solutions have been observed. Since no experimental data are available, it is difficult to determine the reason for this difference. Future works will be oriented towards sensitivity analysis for assessing the influence of initial conditions and empirical coefficients in order to assess the variability of the numerical solution. Other actions will be focused on the extension of the present methods to multi-dimensional problems.

\section{Acknowledgement}



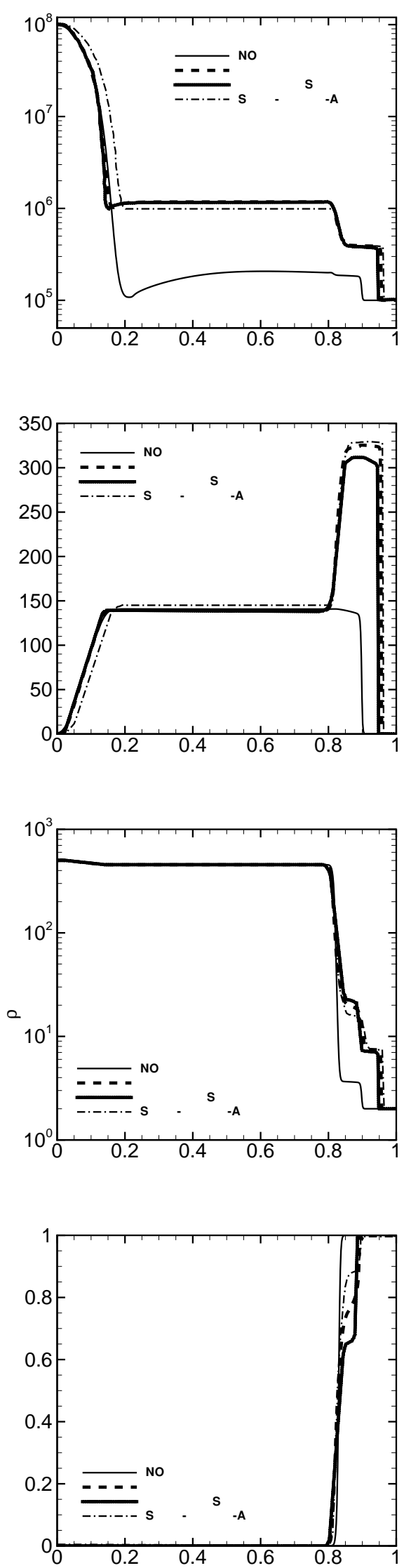

Figure 4: Pressure, velocity, mixture density and $Y=\alpha_{1} \rho_{1} / \rho$ obtained at a 


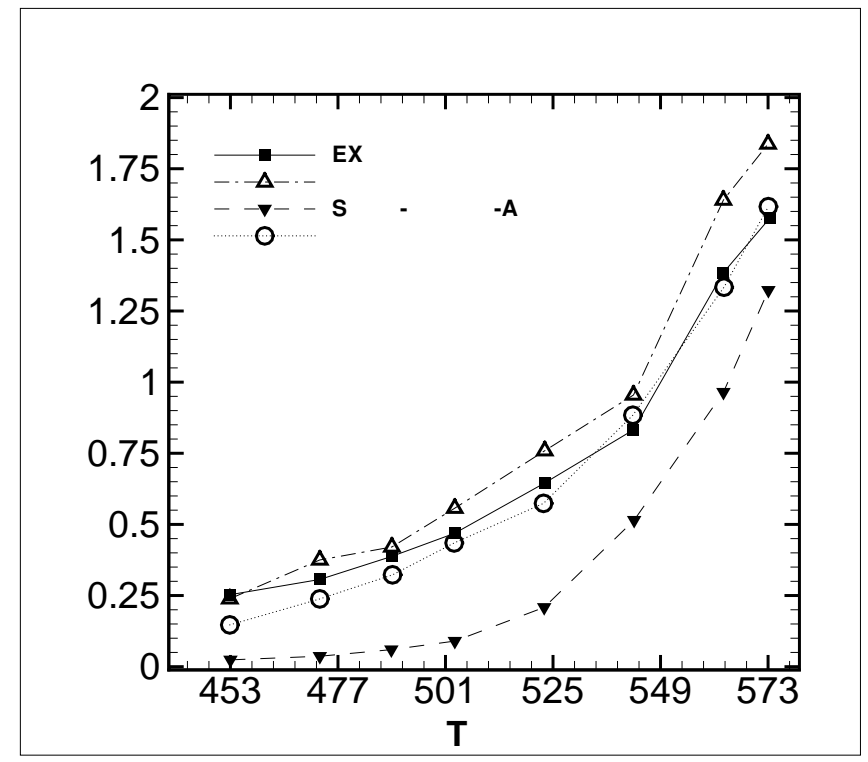

Figure 5: Comparison of the evaporation front velocity obtained for different initial left liquid temperature. The profiles have been obtained experimentally, by the present model, in Saurel et al. [19] and Zein et al. [20. 

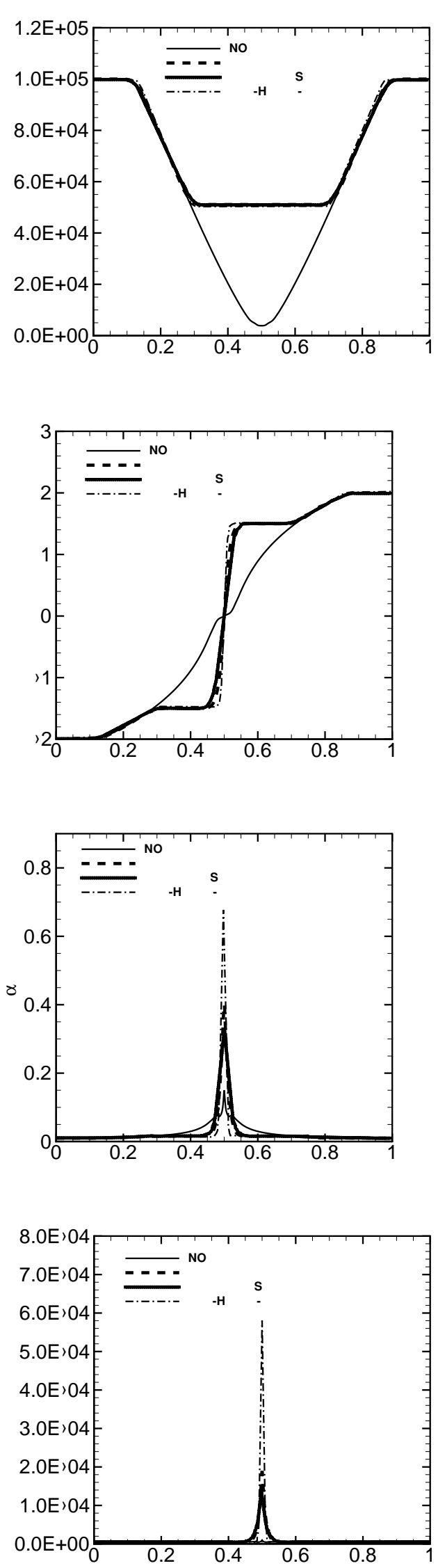

Figure 6: Pressure profile, velocity profile, vapor volume fraction and vapor 

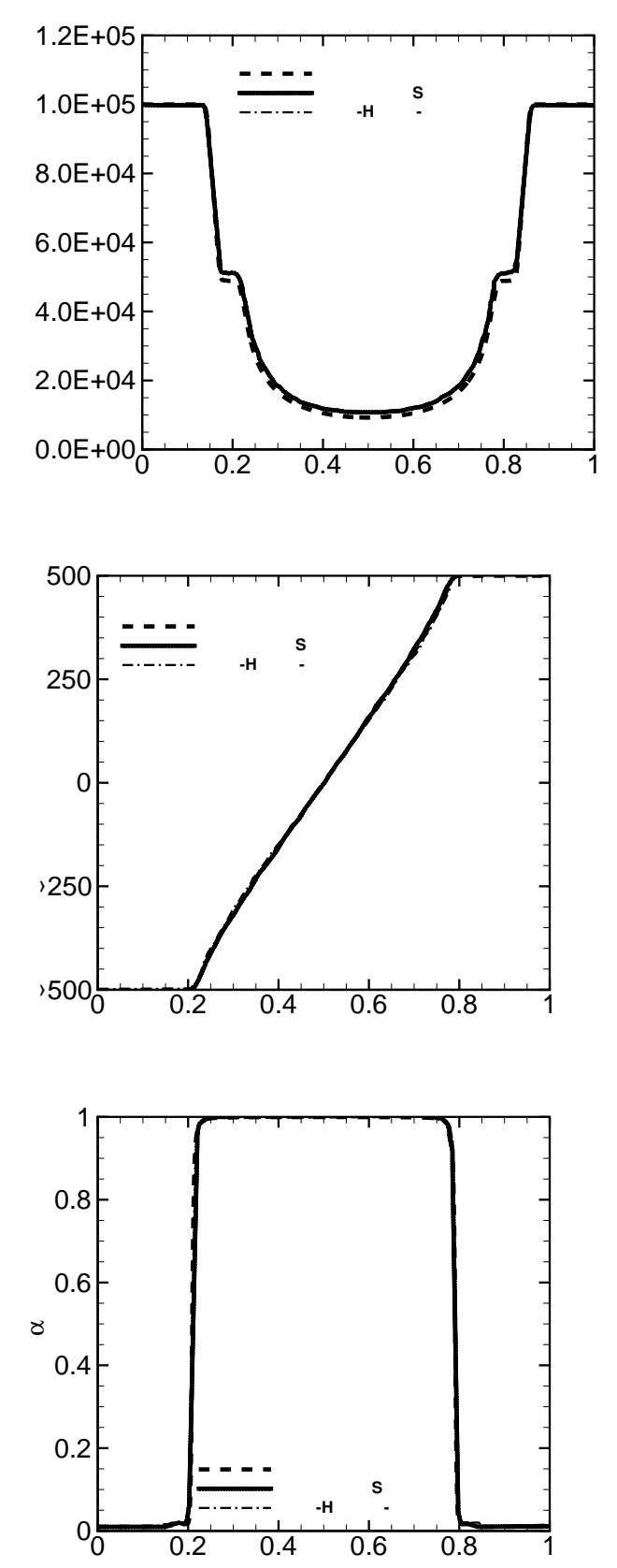

$\mathrm{RR} \mathrm{n}^{\circ} 8664$

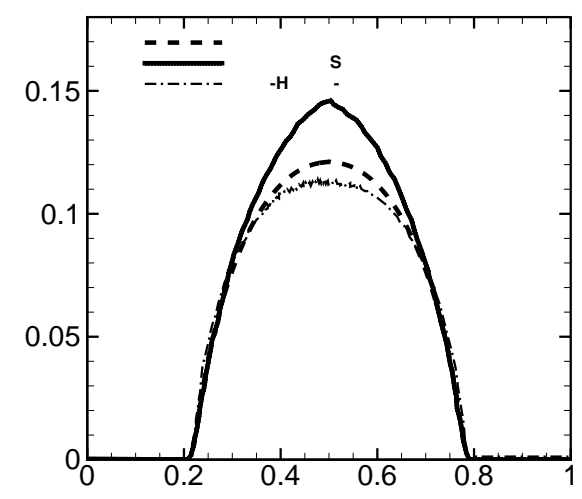

Figure 7: Pressure profile, velocity profile, vapor volume fraction and vapor 


\section{References}

[1] C.E. Brennen. Cavitation and Bubble Dynamics. Oxford University Press, 1995.

[2] R. Saurel, F. Petitpas, and R. Abgrall. Modelling phase transition in metastable liquids: application to cavitating and flashing flows. Journal of Fluid Mechanics, 607:313-350, 2008.

[3] J.P. Franc and C. Pellone. Analysis of thermal effects in a cavitating inducer using rayleigh equation. Journal of Fluids Engineering, 129:974-983, 2007.

[4] M.G. Rodio, M.G. De Giorgi, and A. Ficarella. Influence of convective heat transfer modeling on the estimation of thermal effects in cryogenic cavitating flows. International Journal of Heat and Mass Transfer, 55:65386554, 2012.

[5] M.G. De Giorgi, M.G. Rodio, and A. Ficarella. Thermodynamic effects on cavitation in water and cryogenic fluids. In ASME 2010 10th Biennial Conference on Engineering Systems Design and Analysis, pages 475-482. American Society of Mechanical Engineers, 2010.

[6] A.T. Preston, T. Colonius, and C.E. Brennen. A reduced-order model of diffusive effects on the dynamics of bubbles. Physics of Fluids, 19:123302123313, 2007.

[7] Jintao Liu, Shuhong Liu, Yulin Wu, Lei Jiao, Leqin Wang, and Yuekun Sun. Numerical investigation of the hump characteristic of a pumpturbine based on an improved cavitation model. Computers \& Fluids, 68(0):105 $111,2012$.

[8] E. Goncalves and R. Fortes Patella. Constraints on equation of state for cavitating flows with thermodynamic effects. Applied Mathematics and Computation, 217:5095-5102, 2011.

[9] M.G. De Giorgi, M.G. Rodio, and A. Ficarella. Cavitation modeling in cryogenic fluids for liquid rocket engine applications. AIAA 38th Fluid Dynamics Conference and Exhibit, Seattle, WA, Jun, pages 23-26, 2008.

[10] M.G. Rodio and P.M. Congedo. Robust analysis of cavitating flows in the venturi tube. European Journal of Mechanics-B/Fluids, 44:88-99, 2014.

[11] M.G. De Giorgi, P.M. Congedo, M.G. Rodio, and A. Ficarella. Shape optimization for cryogenic cavitating flows past an isolated hydrofoil. In ASME 2008 Fluids Engineering Division Summer Meeting collocated with the Heat Transfer, Energy Sustainability, and 3rd Energy Nanotechnology Conferences, pages 75-85. American Society of Mechanical Engineers, 2008.

[12] M.R. Baer and J.W. Nunziato. A two-phase mixture theory for deflagration to detonation transition (ddt) in reactive granular materials. International Journal of Multhiphase Flow, 12:861-889, 1986.

[13] R. A. Berry, R. Saurel, and O. LeMetayer. The discrete equation method (dem) for fully compressible, two-phaseflows in ducts of spatially varying cross-section. Nuclear Engineering and Design, 240:3797-3818, 2010. 
[14] M.G. Rodio, P.M. Congedo, and R. Abgrall. Two-phase flow numerical simulation with real-gas effects and occurrence of rarefaction shock waves. European Journal of Mechanics-B/Fluids, 45:20-35, 2014.

[15] A. Murrone and H. Guillard. A five equation reduced model for compressible two-phase flow problems. Journal of Computational Physics, 202:664$698,2005$.

[16] E. Goncalves. Modeling for non isothermal cavitation using 4-equation models. International Journal of Heat and Mass Transfer, 76:247-262, 2014.

[17] R. Saurel and O. LeMetayer. A multiphase model for compressible flows with interfaces, shocks, detonation waves and cavitation. Journal of Fluid Mechanics, 431:239-271, 2001.

[18] R. Abgrall and M.G. Rodio. Discrete equation method (dem) for the simulation of viscous, compressible, two-phase flows. Computers \&3 Fluids, 91(0):164 - 181, 2014.

[19] R. Saurel, F. Petitpas, and R. Abgrall. Modelling phase transition in metastable liquids. application to cavitating and flashing flows. Journal of Fluids Mechanics, 607:313-350, 2008.

[20] A. Zein, M. Hantke, and G. Warnecke. Modeling phase transition for compressible two-phase flows applied to metastable liquids. Journal of Computational Physics, 229:2964-2998, 2010.

[21] M. Pelanti and K. M. Shyue. A mixture-energy-consistent six-equation twophase numerical model for fluids with interfaces, cavitation and evaporation waves. Journal of Computational Physics, 259:331-357, 2014.

[22] F. Daude, P. Galon, Z. Gao, and E. Blaud. Numerical experiments using a hllc-type scheme with $\{$ ALE $\}$ formulation for compressible two-phase flows five-equation models with phase transition. Computers \& Fluids, 94(0):112 $-138,2014$.

[23] Yue Wang, Lu Qiu, Rolf D. Reitz, and Ramachandra Diwakar. Simulating cavitating liquid jets using a compressible and equilibrium two-phase flow solver. International Journal of Multiphase Flow, 63(0):52 - 67, 2014.

[24] R. Abgrall, M.G. Rodio, and P.M. Congedo. Towards an efficient simulation of cavitating flows with real gas effects and uncertainty quantification. In WCCM XI-ECCM V-ECFD VI, Barcelona, Spain, pages 4779-4790, 2014.

[25] G. Faccanoni, S. Kokh, and G. Allaire. Approximation of liquidvapor phase transition for compressible fluids with tabulated $\{\mathrm{EOS}\}$. Comptes Rendus Mathematique, 348(78):473 - 478, 2010.

[26] R. Abgrall and V. Perrier. Asymptotic expansion of multiscale numerical scheme for compressible multiscale flow. SIAM Journal on Scientific Computing, 5:84-115, 2006. 
[27] R. Saurel and R. Abgrall. A multiphase godunov method for compressible multifluid and multiphase flows. Journal of Computational Physics, 150:425-467, 1999.

[28] A. K. Kapila, R. Menikoff, J. B. Bdzil, S. F. Son, and D. S. Stewart. Two-phase modeling of deflagration-to-detonation transition in granular materials: Reduced equations. Physics of Fluids, 13:3002-3024, 2001.

[29] R. Abgrall and R. Saurel. Discrete equations for physical and numerical compressible multiphase mixtures. Journal of Computational Physics, 186:361-396, 2003.

[30] S.K. Godunov. A difference scheme for numerical computation of discontinuous solutions of equations of fluid dynamics. Math. Sbornik, 47:271-306, 1959.

[31] F. Coquel and B. Perthame. Relaxation of energy and approximate riemann solvers for general pressure laws in fluid dynamics. Society for Industrial and Applied Mathematics, 35:2223-2249, 1998.

[32] E.F. Toro. Riemann Solvers and Numerical Methods for Fluid Dynamics. Springer, Berlin, 1997.

[33] G. Faccanoni, S. Kokh, and G. Allaire. Modelling and simulation of liquidvapor phase transition in compressible flows based on thermodynamical equilibrium. ESAIM:M2AN, 46:1029-1054, 2012.

[34] E. Goncalves and R.F. Patella. Numerical simulation of cavitating flows with homogeneous models. Computers and Fluids, 38:1682-1696, 2009.

[35] R. Saurel, O. Le Metayer, J.Massoni, and S. Gavrilyuk. Shock jump relations for multiphase mixtures with stiff mechanical relaxation . shock waves. Shock Waves, 16(3):209-232, 2007.

[36] A. Harten, P.D. Lax, C.D. Levermore, and W. Morokoff. Convex entropies and hyperbolicity for general euler equations. SIAM Journal of Numerical Analysis, 35(6):2117-2127, 1998.

[37] A. Ambroso, C. Chalons, and P.-A. Raviart. A godunov-type method for the seven-equation model of compressible two-phase flow. Computers and Fluids, 54:67-91, 2012.

[38] R. Saurel and O. Lemetayer. A multiphase model for compressible flows with interfaces, shocks, detonation waves and cavitation. Journal of Fluid Mechanics, 431:239-271, 2001.

[39] J.R. Simoes-Moreira and J.E. Shepherd. Evaporation waves in superheated dodecane. J. Fluid Mech., 382:6386, 1999. 


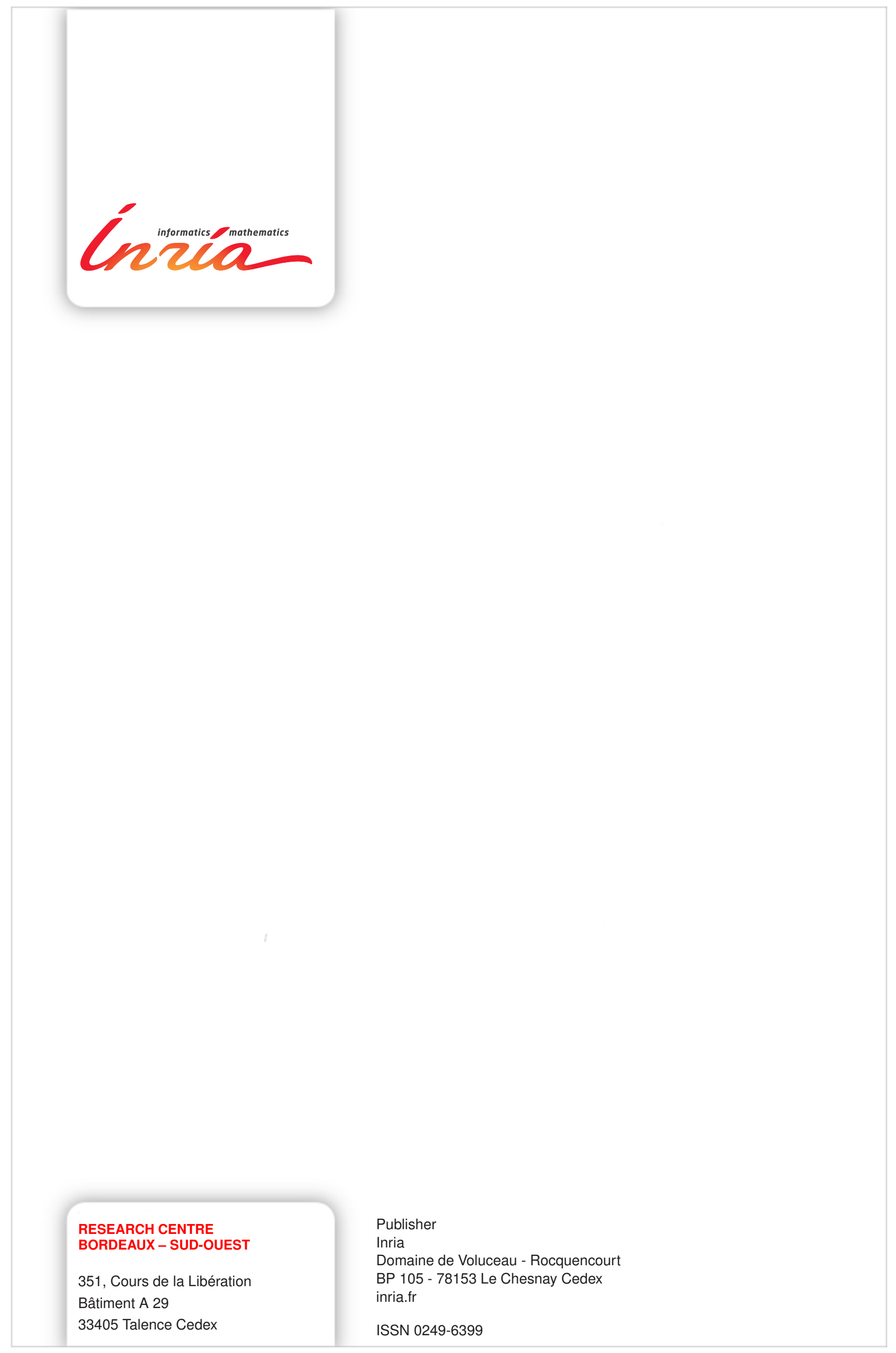

